Research Article

\title{
Trajectory Tracking Control for Small-Scale Unmanned Helicopters with Mismatched Disturbances Based on a Continuous Sliding Mode Approach
}

\author{
Xing Fang $\mathbb{D}^{1}$ and Yujia Shang ${ }^{2}$ \\ ${ }^{1}$ Key Laboratory of Advanced Process Control for Light Industry of the Ministry of Education, Institute of Automation, \\ Jiangnan University, Wuxi 214122, China \\ ${ }^{2}$ Instrumentation Technology and Economy Institute, Beijing 100055, China
}

Correspondence should be addressed to Xing Fang; xingfang@jiangnan.edu.cn

Received 29 October 2018; Revised 21 January 2019; Accepted 11 February 2019; Published 22 April 2019

Academic Editor: Jacopo Serafini

Copyright (C) 2019 Xing Fang and Yujia Shang. This is an open access article distributed under the Creative Commons Attribution License, which permits unrestricted use, distribution, and reproduction in any medium, provided the original work is properly cited.

\begin{abstract}
A novel continuous sliding mode control (CSMC) strategy based on the finite-time disturbance observer (FTDO) is proposed for the small-scale unmanned helicopters in the presence of both matched and mismatched disturbances. First, a novel sliding surface is designed based on the estimates of the mismatched disturbances and their derivatives obtained by the FTDO. Then, a continuous sliding mode control law is developed, which does not lead to any chattering phenomenon. Furthermore, the closed-loop helicopter system is proved to be asymptotically stable. Finally, the excellent hovering and tracking performance, as well as the powerful disturbance rejection capability of the proposed novel CSMC method, is validated by the simulation results.
\end{abstract}

\section{Introduction}

With the prominent capabilities of vertical take-off and landing and hovering and high levels of maneuverability, smallscale unmanned helicopters have become the most popular unmanned aerial vehicles (UAVs). Furthermore, unmanned helicopters can be applied to military and civilian areas. However, unmanned helicopters are known as nonlinear systems with strong couplings and a variety of disturbances. Particularly, some disturbances enter the unmanned helicopter systems via different channels with control inputs, which are known as mismatched disturbances. Therefore, it has become a quite attractive and challenging task to design high-performance control strategies for unmanned helicopters [1-4].

In the past decades, numerous elegant control methods have been developed for the helicopter systems. Some flight controllers are designed based on the linear model of the unmanned helicopters, including PID [5], LQR [6], and $H_{\infty}$ [7]. However, these linear controllers are only effective when the states of the unmanned helicopters are in the neighbourhood of the equilibrium points [8]. Consequently, a growing number of nonlinear control approaches have been employed for the unmanned helicopter systems. By selecting the meaningful outputs of position and yaw angle, the approximate input-output linearization techniques have been proposed for the unmanned helicopters, which are able to take full advantage of the nonlinear dynamics and are applicable to a wide range of flight envelopes [9]. In combination with the adaptive technique, the backstepping control methods have been employed to handle the parametric uncertainties [10]. With the unique disturbance suppression capability, sliding mode control (SMC) methods have been proposed to attenuate the model uncertainties and external disturbances of the unmanned helicopters [11]. However, all the control methods aforementioned are feedback control 
techniques, which are one-degree-of-freedom control structures. The feedback action is responsible both for suppressing the uncertainties and for stabilizing the system. Therefore, a tradeoff will be made for these two performances. Thus, these feedback control methods may not have enough capability to deal with the severe uncertainties and disturbances of the unmanned helicopter systems.

In recent years, the disturbance observer-based control (DOBC) methods have been proposed to handle the uncertainties and disturbances [12-15]. The DOBC methods are considered two-degree-of-freedom control structures. It is composed of two parts: a traditional feedback control loop to meet the requirements on stability and tracking performance for the system and an inner disturbance rejection loop to compensate for the disturbances straightforwardly. The DOBC methods have found applications in a wide range, such as robotic manipulator systems [16], disk drive systems [17], air-breathing supersonic vehicle systems [18], and fluidized bed combustor systems [19]. However, most of the DOBC methods are only able to deal with the disturbances satisfying the matching condition which implies that the disturbances enter the system via the same channels as the control inputs [20].

Nowadays, the DOBC methods have been extended to deal with the systems subjected to mismatched disturbances [21-23]. By tactfully designing a mismatched disturbance compensation gain, a new DOBC algorithm is developed to eliminate the influence of mismatched disturbances from the outputs [24]. A new sliding mode control method is developed to reject the mismatched disturbances [25]. Moreover, the coordinate transformation technique is employed to transform the mismatched disturbances into matched disturbances which can be compensated by a feedforward control technique [26]. However, these control algorithms can only attenuate the mismatched disturbances that go to constants in a steady state. Nevertheless, the mismatched disturbances applied to the helicopter systems are high-order time-varying functions. A disturbance observer-based sliding mode control algorithm is designed to suppress the highorder time-varying mismatched disturbances [27]. However, this control algorithm can only be applied to single-input single-output (SISO) systems. The unmanned helicopters are typical multiple-input multiple-output (MIMO) systems. In article [28], an extended nonlinear disturbance observerbased sliding mode control (ENDO-SMC) algorithm is proposed for the helicopter systems, which is able to attenuate the mismatched disturbances effectively. However, the tracking errors of the helicopter systems are only guaranteed to converge to a neighbourhood of origin, instead of the origin. In addition, the ENDO-SMC algorithm cannot fully eliminate the chattering phenomenon. In this paper, a novel continuous sliding mode control (CSMC) algorithm based on the finite-time disturbance observer (FTDO) is developed for the small-scale unmanned helicopter systems. By designing a new sliding surface with the estimates of the mismatched disturbances and their derivatives by FTDOs, the novel CSMC method is able to suppress both the matched and mismatched disturbances. Furthermore, a new continuous sliding mode control law is designed for the helicopter system, which does not result in any chattering phenomenon. Additionally, it is proved that the novel CSMC method can drive the outputs of the unmanned helicopters to the set-point asymptotically in spite of the presence of both matched and mismatched disturbances. Finally, numerical simulation results demonstrate the effectiveness of the proposed CSMC method.

Compared to other applications of sliding mode control (SMC) for UAVs, the contributions of this manuscript are given as follows. Owing to the fact that the SMC methods are only robust to matched disturbances, most of the SMCbased flight controllers of UAVs cannot suppress the mismatched disturbances that are prominent in UAV systems $[29,30]$. What is worse, due to their discontinuous control actions, the control systems are subject to a chattering phenomenon. An integral sliding mode controller is developed for the unmanned helicopter system to attenuate the mismatched disturbances [31]. Unfortunately, the integral action will bring some adverse effects to the control systems, such as large overshoot and long settling time. In this paper, the proposed novel continuous sliding mode control (CSMC) method is able not only to reject the mismatched disturbances of the unmanned helicopter systems but also to eliminate the chattering phenomenon completely.

Additionally, the neural networks also have been employed for the flight controller to reject mismatched disturbances widely. A nonlinear adaptive neural networkbased controller is designed for unmanned helicopter systems, whose tracking error can be restricted within a small bound [32]. Moreover, a neural network-based adaptive sliding mode tracking controller is developed for unmanned helicopter systems, which is able to compensate for the external unknown disturbances [33]. With the combination of the neural networks and backstepping technique, both these two flight controllers can deal with mismatched disturbances. Unfortunately, an exact helicopter model is needed a priori, which compromises the feasibility of these methods. On the other hand, a great deal of training data is required for the neural networks, which is the general problem of the neural network technique.

This paper is organized as follows. The dynamic model of the small-scale unmanned helicopter is given in Section 2. Section 3 presents the complete design procedure of the proposed CSMC method. The stability analysis of the closedloop helicopter system is given in Section 4. Some simulation results that demonstrate the effectiveness of the proposed CSMC method are illustrated in Section 5. Section 6 draws the conclusions.

\section{Problem Formulation}

2.1. Nonlinear Dynamic Model of the Unmanned Helicopter. This section presents the nonlinear dynamic model of the unmanned helicopter. The unmanned helicopter is considered a six-degree-of-freedom rigid body model with a simplified force and moment generation process and disturbed by external disturbances and model uncertainties, which are treated as the lumped disturbances. The schematic diagram of the unmanned helicopter is shown in Figure 1. 


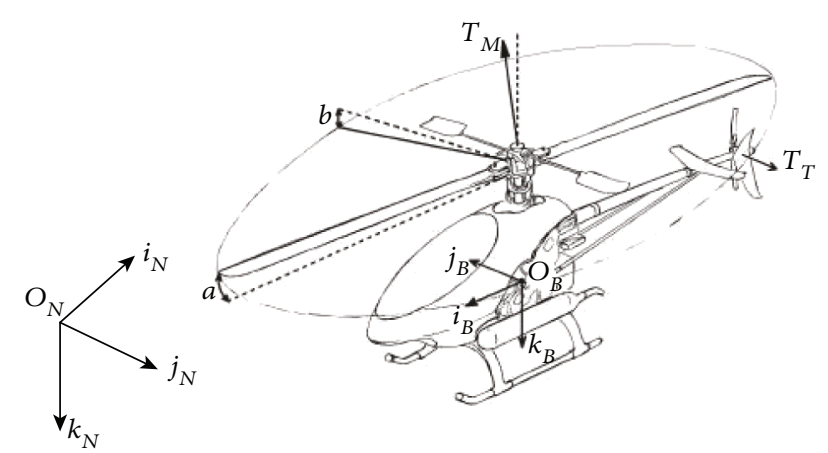

FIGURE 1: Schematic diagram of a small-scale unmanned helicopter.

The nonlinear dynamic model of a small-scale unmanned helicopter can be presented in the following form $[7,34]$ :

$$
\begin{aligned}
& \dot{P}=V, \\
& \dot{V}=g e_{3}+R(\Theta) e_{3}\left(-g+Z_{w} w+Z_{\mathrm{col}} \delta_{\mathrm{col}}\right)+f_{d}, \\
& \dot{\Theta}=\prod(\Theta) \Omega, \\
& \dot{\Omega}=-J^{-1} \Omega \times J \Omega+A \Omega+B u+\tau_{d},
\end{aligned}
$$

where $P=\left[\begin{array}{lll}x & y & z\end{array}\right]^{T}$ and $V=\left[\begin{array}{lll}u & v & w\end{array}\right]^{T}$ represent the position and velocity vectors in the inertial frame and $\Theta=\left[\begin{array}{lll}\phi & \theta & \psi\end{array}\right]^{T}$ and $\Omega=\left[\begin{array}{lll}p & q & r\end{array}\right]^{T}$ represent the Euler angle and angular rate vectors in the body-fixed frame. $u=\left[\begin{array}{llll}\delta_{\text {col }} & \delta_{\text {lon }} & \delta_{\text {lat }} & \delta_{\text {ped }}\end{array}\right]^{T}$ is the control input vector. The notations of $f_{d}$ and $\tau_{d}$ are the lumped disturbance vectors acting on the unmanned helicopter, which contain wind gusts, model uncertainties, and so on.

The notation of $g$ denotes the gravitational acceleration, $e_{3}=\left[\begin{array}{lll}0 & 0 & 1\end{array}\right]^{T}$ is a unitary vector, and $J=\operatorname{diag}\left(J_{x x}, J_{y y}\right.$, $J_{z z}$ ) is the diagonal inertia matrix. $Z_{w}$ and $Z_{\text {col }}$ are the stability derivative and input derivative of the main rotor thrust $T_{m}=m\left(-g+Z_{w} w+Z_{\text {col }} \delta_{\text {col }}\right)$, respectively. $A \in \mathbb{R}^{3 \times 3}$ and $B \in \mathbb{R}^{3 \times 4}$ are the stability derivative matrix and input derivative matrix of the moment vector $\tau=A \Omega+B u$, respectively. $R(\Theta)$ and $\Pi(\Theta)$ represent the rotation matrix and attitude kinematic matrix, respectively, defined as

$$
\begin{aligned}
R(\Theta) & =\left[\begin{array}{ccc}
C_{\theta} C_{\psi} & S_{\phi} S_{\theta} C_{\psi}-C_{\phi} S_{\psi} & C_{\phi} S_{\theta} C_{\psi}+S_{\phi} S_{\psi} \\
C_{\theta} S_{\psi} & S_{\phi} S_{\theta} S_{\psi}+C_{\phi} C_{\psi} & C_{\phi} S_{\theta} S_{\psi}-S_{\phi} C_{\psi} \\
-S_{\theta} & S_{\phi} C_{\theta} & C_{\phi} C_{\theta}
\end{array}\right], \\
\prod(\Theta) & =\left[\begin{array}{ccc}
1 & S_{\phi} T_{\theta} & C_{\phi} T_{\theta} \\
0 & C_{\phi} & -S_{\phi} \\
0 & \frac{S_{\phi}}{C_{\theta}} & \frac{S_{\phi}}{C_{\theta}}
\end{array}\right]
\end{aligned}
$$

where the compact notation $C$ denotes $\cos (\cdot), S$ denotes sin $(\cdot)$, and $T$ denotes $\tan (\cdot)$.

2.2. Approximate Feedback Linearized Model of the Unmanned Helicopter. The control task is to design the control input $u=\left[\begin{array}{llll}\delta_{\text {col }} & \delta_{\text {lon }} & \delta_{\text {lat }} & \delta_{\text {ped }}\end{array}\right]^{T}$ for the unmanned helicopter to drive the position and yaw angle to the desired trajectory of $P_{r}=\left[\begin{array}{lll}x_{r}(t) & y_{r}(t) & z_{r}(t)\end{array}\right]^{T}$ and $\psi_{r}(t)$. However, the exact input-output linearization fails to linearize the complete helicopter system, leading to unstable zero dynamics [9]. Therefore, the approximate feedback linearization technique will be developed for the unmanned helicopter system.

Let the lumped disturbances be $f_{d}=\tau_{d}=0$ at first. Furthermore, in order to develop the feedback linearization procedure succinctly, the term $-m\left(-g+Z_{w} w+Z_{\text {col }} \delta_{\text {col }}\right)$ will be replaced by the main rotor thrust $T_{m}$. The simplified nominal model of the unmanned helicopter can be described by

$$
\left\{\begin{array}{l}
\dot{P}=V, \\
\dot{V}=g e_{3}-\frac{1}{m} R(\Theta) e_{3} T_{m}, \\
\dot{\Theta}=\prod(\Theta) \Omega, \\
\dot{\Omega}=-J^{-1} \Omega \times J \Omega+A \Omega+B u .
\end{array}\right.
$$

The dimension of the helicopter system (3) is $n=12$. Meanwhile, the total relative degrees are $r=8$. Therefore, there exists internal dynamics characterized by 4-D zero dynamics [35]. To make the model (3) feedback linearizable, the dynamic extension procedure is proposed for the helicopter system, which is realized by two integrators of the main rotor thrust $T_{m}$.

Furthermore, to simplify the feedback linearization procedure, the following transformation of the control input is proposed:

$$
\tilde{\tau}=-J^{-1} \Omega \times J \Omega+A \Omega+B u
$$

Therefore, we can obtain $\dot{\Omega}=\tilde{\tau}=\left[\begin{array}{lll}\tilde{\tau}_{\phi} & \tilde{\tau}_{\theta} & \tilde{\tau}_{\psi}\end{array}\right]^{T}$. And the following expression is regarded as the new control input vector $\tilde{u}=\left[\begin{array}{llll}\tilde{\tau}_{\phi} & \tilde{\tau}_{\theta} & \tilde{\tau}_{\psi} & \tilde{T}_{m}\end{array}\right]^{T}$, where $\tilde{T}_{m}=\ddot{T}_{m}$.

In accordance with the input-output feedback linearization procedure, we differentiate the output variables $P$ and $\psi$ in (3) until input variables appear in the equation.

The fourth-order derivative of position $P$ can be obtained as

$$
P^{(4)}=f_{P}-\frac{1}{m} R K\left(T_{m}\right)\left[\begin{array}{lll}
\tilde{\tau}_{\phi} & \tilde{\tau}_{\theta} & \tilde{T}_{m}
\end{array}\right]^{T},
$$

where the input variables occur, and 


$$
\begin{aligned}
f_{P} & =-\frac{1}{m} R S^{2} e_{3} T_{m}-\frac{2}{m} R S e_{3} \dot{T}_{m} \\
K\left(T_{m}\right)= & {\left[\begin{array}{ccc}
0 & T_{m} & 0 \\
-T_{m} & 0 & 0 \\
0 & 0 & 1
\end{array}\right], } \\
S= & {\left[\begin{array}{ccc}
0 & -r & q \\
r & 0 & -p \\
-q & p & 0
\end{array}\right] . }
\end{aligned}
$$

The second-order derivative of $\psi$ can be obtained as

$$
\ddot{\psi}=f_{\psi}+\frac{S_{\phi}}{C_{\theta}} \tilde{\tau}_{\theta}+\frac{C_{\phi}}{C_{\theta}} \tilde{\tau}_{\psi},
$$

where the input variables appear, and $f_{\psi}=\left(\left(C_{\phi} / C_{\theta}\right) \dot{\phi}+\right.$ $\left.\left(S_{\phi} S_{\theta} / C_{\theta}^{2}\right) \dot{\theta}\right) q+\left(-\left(S_{\phi} / C_{\theta}\right) \dot{\phi}+\left(C_{\phi} S_{\theta} / C_{\theta}^{2}\right) \dot{\theta}\right) r$.

The dimension of the extended helicopter system is $n_{e}=14$, and the total relative degree is $r_{e}=14$. Therefore, we conclude that there exists no internal dynamics in the extended helicopter system anymore. So it can be completely feedback linearized.

Define the new state variables $\xi_{1}=P, \xi_{2}=\dot{P}, \xi_{3}=\ddot{P}$, $\xi_{4}=P^{(3)}, \xi_{5}=\psi$ and $\xi_{6}=\dot{\psi}$, and consider the disturbances $f_{d}$ and $\tau_{d}$ again. We can derive the complete model of the small-scale unmanned helicopter as

$$
\begin{aligned}
& S_{1}\left\{\begin{array}{l}
\dot{\xi}_{1}=\xi_{2}, \\
\dot{\xi}_{2}=\xi_{3}+d_{1}, \\
\dot{\xi}_{3}=\xi_{4}, \\
\dot{\xi}_{4}=f_{P}-\frac{1}{m} R K\left(T_{m}\right)\left[\begin{array}{lll}
\tilde{\tau}_{\phi} & \tilde{\tau}_{\theta} & \tilde{T}_{m}
\end{array}\right]^{T}+d_{2},
\end{array}\right. \\
& S_{2}\left\{\begin{array}{l}
\dot{\xi}_{5}=\xi_{6}, \\
\dot{\xi}_{6}=f_{\psi}+\frac{S_{\phi}}{C_{\theta}} \tilde{\tau}_{\theta}+\frac{C_{\phi}}{C_{\theta}} \tilde{\tau}_{\psi}+d_{3},
\end{array}\right.
\end{aligned}
$$

where $\quad d_{1}=\left[\begin{array}{lll}d_{11} & d_{12} & d_{13}\end{array}\right]^{T}=(1 / m) R f_{d} \in \mathbb{R}^{3}, \quad d_{2}=\left[\begin{array}{ll}d_{21} & \end{array}\right.$ $\left.d_{22} d_{23}\right]^{T}=(1 / m) R T_{m}\left[\begin{array}{lll}J_{2}^{-1} \tau_{d 2} & -J_{1}^{-1} \tau_{d 1} & 0\end{array}\right]^{T} \in \mathbb{R}^{3}$, and $d_{3}$ $=\left(S_{\phi} / C_{\theta}\right) J_{2}^{-1} \tau_{d 2}+\left(C_{\phi} / C_{\theta}\right) J_{3}^{-1} \tau_{d 3} \in \mathbb{R}$ represent the lumped disturbances acting on the helicopter system. Furthermore, $d_{2}$ and $d_{3}$ enter the helicopter system via the same channels as control inputs, so they are matched disturbances. On the other hand, $d_{1}$ does not satisfy the matching condition, so it is known as mismatched disturbance.

2.3. Control Objective. The objective of this paper is to develop a novel continuous sliding mode controller for the small-scale unmanned helicopter to track the predefined trajectory asymptotically despite the presence of both matched and mismatched disturbances. The proposed novel CSMC strategy can suppress the matched disturbances, as well as the mismatched disturbances. Furthermore, the proposed CSMC method is able to eliminate the chattering phenomenon completely. The flight controller structure is illustrated in Figure 2.

\section{Controller Design}

This section presents a novel continuous sliding mode controller based on the finite-time disturbance observer for the small-scale unmanned helicopter with 190 matched and mismatched disturbances.

3.1. Finite-Time Disturbance Observer (FTDO). First of all, some FTDOs are designed to estimate the disturbances and their successive derivatives in finite time.

To develop the subsequent FTDOs, a reasonable assumption is given as follows.

Assumption 1. The disturbances in the helicopter system ((8) and (9)) satisfy the idea that the mismatched disturbance $d_{1}$ is at least 3-th order differentiable and $d_{1}^{(3)}$ has the Lipschitz constant of $L_{1}$ and the matched disturbances $d_{2}$ and $d_{3}$ are at least 1-th order differentiable and $\dot{d}_{2}$ and $\dot{d}_{3}$ have Lipschitz constants $L_{2}$ and $L_{3}$, respectively.

A third-order FTDO is designed to estimate the mismatched disturbance $d_{1}$ and its derivatives $\dot{d}_{1}$ and $\ddot{d}_{1}$ [36]:

$$
\begin{aligned}
& \dot{z}_{0}^{1}=v_{0}^{1}+\xi_{3}, \\
& v_{0}^{1}=-\lambda_{0}^{1} L_{1}^{1 / 4}\left\|z_{0}^{1}-\xi_{2}\right\|^{3 / 4} \operatorname{sign}\left(z_{0}^{1}-\xi_{2}\right)+z_{1}^{1}, \\
& \dot{z}_{1}^{1}=v_{1}^{1}, \\
& v_{1}^{1}=-\lambda_{1}^{1} L_{1}^{1 / 3}\left\|z_{1}^{1}-v_{0}^{1}\right\|^{2 / 3} \operatorname{sign}\left(z_{1}^{1}-v_{0}^{1}\right)+z_{2}^{1}, \\
& \dot{z}_{2}^{1}=v_{2}^{1}, \\
& v_{2}^{1}=-\lambda_{2}^{1} L_{1}^{1 / 2}\left\|z_{2}^{1}-v_{1}^{1}\right\|^{1 / 2} \operatorname{sign}\left(z_{2}^{1}-v_{1}^{1}\right)+z_{3}^{1}, \\
& \dot{z}_{3}^{1}=v_{3}^{1}, \\
& v_{3}^{1}=-\lambda_{3}^{1} L_{1} \operatorname{sign}\left(z_{3}^{1}-v_{2}^{1}\right),
\end{aligned}
$$

where $z_{0}^{1}$ is the estimate of state $\xi_{2} . z_{1}^{1}, z_{2}^{1}$, and $z_{3}^{1}$ are the estimates of disturbance $d_{1}$ and its successive derivatives $\dot{d}_{1}$ and $\ddot{d}_{2}$, respectively. $L_{1}>0$ and $\lambda_{i}^{1}>0(i=0,1,2,3)$ are the coefficients of the FTDO to be designed.

A first-order FTDO is designed to estimate the matched disturbanced ${ }_{2}$ :

$$
\begin{aligned}
& \dot{z}_{0}^{2}=v_{0}^{2}+\left(f_{P}-\frac{1}{m} R K\left[\begin{array}{lll}
\tilde{\tau}_{\phi} & \tilde{\tau}_{\theta} & \tilde{T}_{m}
\end{array}\right]^{T}\right), \\
& v_{0}^{2}=-\lambda_{0}^{2} L_{2}^{1 / 2}\left\|z_{0}^{2}-\xi_{4}\right\|^{1 / 2} \operatorname{sign}\left(z_{0}^{2}-\xi_{4}\right)+z_{1}^{2}, \\
& \dot{z}_{1}^{2}=v_{1}^{2}, \\
& v_{1}^{2}=-\lambda_{1}^{2} L_{2} \operatorname{sign}\left(z_{1}^{2}-v_{0}^{2}\right),
\end{aligned}
$$




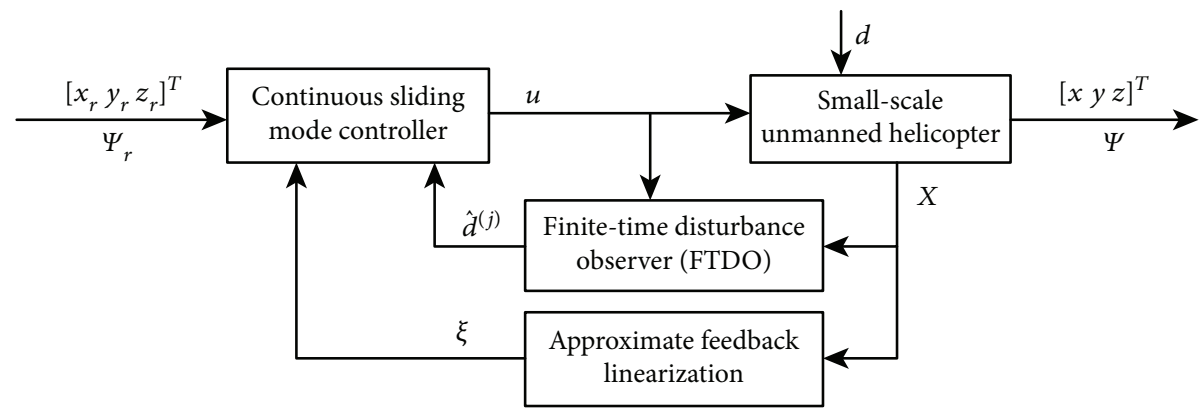

Figure 2: The flight controller structure of a small-scale unmanned helicopter.

where $z_{0}^{2}$ is the estimate of state $\xi_{4}$ and $z_{1}^{2}$ is the estimate of disturbance $d_{2}$.

$L_{2}>0$ and $\lambda_{i}^{2}>0(i=0,1)$ are the coefficients of the FTDO to be designed.

A first-order FTDO is designed to estimate the matched disturbance $d_{3}$ :

$$
\begin{aligned}
& \dot{z}_{0}^{3}=v_{0}^{3}+\left(f_{\psi}+\frac{S_{\phi}}{C_{\theta}} \tilde{\tau}_{\theta}+\frac{C_{\phi}}{C_{\theta}} \tilde{\tau}_{\psi}\right), \\
& v_{0}^{3}=-\lambda_{0}^{3} L_{3}^{1 / 2}\left|z_{0}^{3}-\xi_{6}\right|^{1 / 2} \operatorname{sign}\left(z_{0}^{3}-\xi_{6}\right)+z_{1}^{3}, \\
& \dot{z}_{1}^{3}=v_{1}^{3}, \\
& v_{1}^{3}=-\lambda_{1}^{3} L_{3} \operatorname{sign}\left(z_{1}^{3}-v_{0}^{3}\right),
\end{aligned}
$$

where $z_{0}^{3}$ is the estimate of state $\xi_{6}$ and $z_{1}^{3}$ is the estimate of disturbance $d_{3}$.

$L_{3}>0$ and $\lambda_{i}^{3}>0(i=0,1)$ are the coefficients of the FTDO to be designed.

Combining the helicopter system (8) and the FTDO ((10)-(17)), the error dynamics of the FTDO satisfies the following differential inclusion understood in the Filippov sense:

$$
\begin{aligned}
& \dot{e}_{0}^{1}=-\lambda_{0}^{1} L_{1}^{1 / 4}\left\|e_{0}^{1}\right\|^{3 / 4} \operatorname{sign}\left(e_{0}^{1}\right)+e_{1}^{1}, \\
& \dot{e}_{1}^{1}=-\lambda_{1}^{1} L_{1}^{1 / 3}\left\|e_{1}^{1}-\dot{e}_{0}^{1}\right\|^{2 / 3} \operatorname{sign}\left(e_{1}^{1}-\dot{e}_{0}^{1}\right)+e_{2}^{1}, \\
& \dot{e}_{2}^{1}=-\lambda_{2}^{1} L_{1}^{1 / 2}\left\|e_{2}^{1}-\dot{e}_{1}^{1}\right\|^{1 / 2} \operatorname{sign}\left(e_{2}^{1}-\dot{e}_{1}^{1}\right)+e_{3}^{1}, \\
& \dot{e}_{3}^{1} \in-\lambda_{3}^{1} L_{1} \operatorname{sign}\left(e_{3}^{1}-\dot{e}_{2}^{1}\right)+\left[-L_{1}, L_{1}\right],
\end{aligned}
$$

where the estimation errors are defined by $e_{0}^{1}=z_{0}^{1}-\xi_{2}$, $e_{1}^{1}=z_{1}^{1}-d_{1}, e_{2}^{1}=z_{2}^{1}-\dot{d}_{1}$, and $e_{3}^{1}=z_{3}^{1}-\ddot{d}_{1}$. It follows from $[36,37]$ that the estimation errors $e_{i}^{1}(t)(i=0,1,2,3)$ will converge to the origin in finite time. That is to say, there exists a time constant $t_{f}$ such that $e_{i}^{1}(t)=0$ for $t>t_{f}$. Moreover, after a finite time $t_{f}$, the following equality is true $z_{i}^{1}=v_{i-1}^{1}(i=1,2,3)$.

The stability analyses of the FTDOs ((18)-(21) and (22) -(25)) are similar to that of the FTDO ((10)-(17)) above. Therefore, it is omitted here for space reason.
3.2. Novel Continuous Sliding Mode Controller. Define the tracking errors and their successive derivatives of position and yaw angle:

$$
\begin{aligned}
& e_{\xi n}=\xi_{n}-P_{r}^{(n-1)}, \quad n=1,2,3,4, \\
& e_{\psi n}=\xi_{n+4}-\psi_{r}^{(n-1)}, \quad n=1,2,
\end{aligned}
$$

where $P_{r}^{(n-1)}$ and $\psi_{r}^{(n-1)}$ are the successive derivatives of the desired position and yaw angle.

The error dynamics of the unmanned helicopter system can be obtained as

$$
S_{1}\left\{\begin{array}{l}
\dot{e}_{\xi 1}=e_{\xi 2}, \\
\dot{e}_{\xi 2}=e_{\xi 3}+d_{1}, \\
\dot{e}_{\xi 3}=e_{\xi 4}, \\
\dot{e}_{\xi 4}=f_{P}-\frac{1}{m} R K\left(T_{m}\right)\left[\begin{array}{lll}
\tilde{\tau}_{\phi} & \tilde{\tau}_{\theta} & \tilde{T}_{m}
\end{array}\right]^{T}-P_{r}^{(4)}+d_{2},
\end{array}\right.
$$

$$
S_{2}\left\{\begin{array}{l}
\dot{e}_{\psi 1}=e_{\psi 2}, \\
\dot{e}_{\psi 2}=f_{\psi}+\frac{S_{\phi}}{C_{\theta}} \tilde{\tau}_{\theta}+\frac{C_{\phi}}{C_{\theta}} \tilde{\tau}_{\psi}-\psi_{r}^{(2)}+d_{3} .
\end{array}\right.
$$

The disturbance $d_{1}$ is mismatched disturbance, while the disturbances $d_{2}$ and $d_{3}$ are matched disturbances. However, the traditional sliding mode control method is only robust to matched disturbances, but sensitive to mismatched disturbances. Therefore, this section will develop a novel continuous sliding mode controller for the helicopter system, which can handle both matched and mismatched disturbances. In addition, it is able to remove the chattering phenomenon completely.

3.2.1. Position Control. The position subsystem (28) is subjected to mismatched disturbance $d_{1}$ and matched disturbance $d_{2}$ simultaneously. Thus, a novel sliding surface based on the FTDO is designed as

$$
\sigma_{P}=\left(e_{\xi 4}+z_{2}^{1}\right)+\Lambda_{3}\left(e_{\xi 3}+z_{1}^{1}\right)+\Lambda_{2} e_{\xi 2}+\Lambda_{1} e_{\xi 1},
$$

where $\Lambda_{i}=\operatorname{diag}\left(\lambda_{i 1}, \lambda_{i 2}, \lambda_{i 3}\right), i=1,2,3$ represent the positive definite diagonal matrices and their elements make $P_{j}(s)=$ 
$s^{3}+\lambda_{3, j} s^{2}+\lambda_{2, j} s+\lambda_{1, j}, j=1,2,3$ be Hurwitz polynomials. The expressions of $z_{1}^{1}$ and $z_{2}^{1}$ are the estimates of the mismatched disturbance $d_{1}$ and its derivative $d_{1}^{(1)}$, respectively.

The time derivative of the sliding surface (30) along the position dynamics (28) can be derived by

$$
\begin{aligned}
\dot{\sigma}_{P}= & \left(f_{P}-\frac{1}{m} R K\left[\begin{array}{lll}
\tilde{\tau}_{\phi} & \tilde{\tau}_{\theta} & \tilde{T}_{m}
\end{array}\right]^{T}-P_{r}^{(4)}+d_{2}+v_{2}^{1}\right) \\
& +\Lambda_{3}\left(e_{\xi 4}+v_{1}^{1}\right)+\Lambda_{2}\left(e_{\xi 3}+d_{1}\right)+\Lambda_{1} e_{\xi 2} .
\end{aligned}
$$

The control input can be designed as

$$
\begin{aligned}
{\left[\begin{array}{ccc}
\tilde{\tau}_{\phi} & \tilde{\tau}_{\theta} & \tilde{T}_{m}
\end{array}\right]^{T}=} & m K^{-1} R^{T}\left(f_{P}-P_{r}^{(4)}+z_{1}^{2}+v_{2}^{1}\right. \\
& +\Lambda_{3}\left(e_{\xi 4}+v_{1}^{1}\right)+\Lambda_{2}\left(e_{\xi 3}+z_{1}^{1}\right) \\
& \left.+\Lambda_{1} e_{\xi 2}+K_{P} \frac{\sigma_{P}}{\left\|\sigma_{P}\right\|^{1-\alpha_{1}}}\right)
\end{aligned}
$$

where $K_{P}=\operatorname{diag}\left(k_{p 1}, k_{p 2}, k_{p 3}\right)$ is the positive definite diagonal matrix and $0<\alpha_{1}<1$.

3.2.2. Yaw Angle Control. Since the yaw angle subsystem (29) is only subjected to matched disturbance $d_{3}$, a traditional sliding surface is chosen as

$$
\sigma_{\psi}=e_{\psi 2}+\lambda_{4} e_{\psi 1}
$$

where $\lambda_{4}>0$.

The time derivative of the sliding surface (33) along the yaw dynamics (29) can be obtained as

$$
\dot{\sigma}_{\psi}=f_{\psi}+\frac{S_{\phi}}{C_{\theta}} \tilde{\tau}_{\theta}+\frac{C_{\phi}}{C_{\theta}} \tilde{\tau}_{\psi}-\ddot{\psi}_{r}+d_{3}+\lambda_{4} e_{\psi 2}
$$

The control input can be designed as

$$
\begin{aligned}
\tilde{\tau}_{\psi}= & -\frac{C_{\theta}}{C_{\phi}}\left(f_{\psi}+\frac{S_{\phi}}{C_{\theta}} \tilde{\tau}_{\theta}-\ddot{\psi}_{r}+z_{1}^{3}+\lambda_{4} e_{\psi 2}\right. \\
& \left.+K_{\psi}\left|\sigma_{\psi}\right|^{\alpha_{2}} \operatorname{sign}\left(\sigma_{\psi}\right)\right),
\end{aligned}
$$

where $K_{\psi}>0$ and $0<\alpha_{2}<1$.

\section{Stability Analysis}

The stability of the closed-loop helicopter system will be analyzed in this section.

Theorem 1. For position subsystem (8) with the sliding surface (30) under the novel continuous sliding mode control law (32), the position $P$ of the unmanned helicopter will converge to the desired trajectory asymptotically and all the states remain bounded despite the presence of matched disturbance $d_{2}$ and mismatched disturbance $d_{1}$.
Proof 1. This proof will be given in three steps.

In the first step, we will show that the bounded estimation errors $e_{i}^{j}$ of FTDO will not make the sliding variable $\sigma_{P}$ go to infinity in any finite time. That is to say, the transition process of FTDO does not destabilize the sliding mode dynamics controlled by observer-based control laws.

With the continuous sliding mode control law (32), the closed-loop position dynamics can be described by

$$
\dot{\sigma}_{P}=\left(d_{2}-z_{1}^{2}\right)-K_{P} \frac{\sigma_{P}}{\left\|\sigma_{P}\right\|^{1-\alpha_{1}}}=-e_{1}^{2}-K_{P} \frac{\sigma_{P}}{\left\|\sigma_{P}\right\|^{1-\alpha_{1}}},
$$

where $e_{1}^{2}=z_{1}^{2}-d_{2}$ is the estimation error of disturbance $d_{2}$, which is bounded due to the finite-time stability of the FTDO.

Let us define a finite-time bounded (FTB) function [38] as

$$
V_{1}\left(\sigma_{P}\right)=\frac{1}{2} \sigma_{P}^{T} \sigma_{P}
$$

Considering the sliding mode dynamics (36), the time derivative of (37) can be obtained as

$$
\begin{aligned}
\dot{V}_{1}\left(\sigma_{P}\right) & =\sigma_{P}^{T} \dot{\sigma}_{P}=\sigma_{P}^{T}\left(-e_{1}^{2}-K_{P} \frac{\sigma_{P}}{\left\|\sigma_{P}\right\|^{1-\alpha_{1}}}\right) \\
& \leq-\lambda_{\max }\left(K_{P}\right)\left\|\sigma_{P}\right\|^{1+\alpha_{1}}-\sigma_{P}^{T} e_{1}^{2} \leq-\sigma_{P}^{T} e_{1}^{2} \\
& \leq \frac{1}{2} \sigma_{P}^{T} \sigma_{P}+\frac{1}{2} e_{1}^{2^{T}} e_{1}^{2} \leq K_{v 1} V_{1}\left(\sigma_{P}\right)+L_{v 1},
\end{aligned}
$$

where $K_{v 1}=1, L_{v 1}=(1 / 2) e_{1}^{2^{T}} e_{1}^{2}$, and $\lambda_{\max }\left(K_{P}\right)$ represents the maximum eigenvalue of diagonal matrix $K_{P}$.

Therefore, we can obtain the FTB function $V_{1}\left(\sigma_{P}\right)$, and so the sliding variable $\sigma_{P}$ will not tend to infinity in any finite time.

Furthermore, the FTDO is finite-time stable, so the estimation error $e_{1}^{2}$ will go to zero in a finite time $t_{f}$. Therefore, combining the fact that the sliding variable $\sigma_{P}$ will not tend to infinity in any finite time, we can obtain that the sliding mode dynamics (36) will reduce to

$$
\dot{\sigma}_{P}=-K_{P} \frac{\sigma_{P}}{\left\|\sigma_{P}\right\|^{1-\alpha_{1}}}
$$

when $t>t_{f}$.

Hence, the sliding variable $\sigma_{P}$ will converge to zero in a finite time $t_{\sigma}$, which means that the transition process of FTDO does not destabilize the sliding mode dynamics controlled by observer-based control laws.

In the second step, we will show that the bounded estimation errors $e_{i}^{j}$ and bounded sliding variable $\sigma_{P}$ will not make the variables $\bar{e}_{\xi 1}, \bar{e}_{\xi 2}, \bar{e}_{\xi 3}$, and $\bar{e}_{\xi_{4}}$ go to infinity in any finite time. That is to say, the transition process of FTDO and the transition process of sliding mode dynamics do not destabilize the closed-loop system controlled by observer-based control laws. 
The new state variables are defined by $\bar{e}_{\xi_{1}}=e_{\xi_{1}}, \bar{e}_{\xi_{2}}=e_{\xi_{2}}$, $\bar{e}_{\xi_{3}}=e_{\xi_{3}}+z_{1}^{1}$, and $\bar{e}_{\xi_{4}}=e_{\xi_{4}}+z_{2}^{1}$. The sliding surface can be rewritten by $\sigma_{P}=\bar{e}_{\xi 4}+\Lambda_{3} \bar{e}_{\xi 3}+\Lambda_{2} \bar{e}_{\xi_{2}}+\Lambda_{1} \bar{e}_{\xi_{1}}$. Therefore, the dynamics of the closed-loop system can be described by

$$
\begin{aligned}
\dot{\bar{e}}_{\xi 1} & =\bar{e}_{\xi 2}, \\
\dot{\bar{e}}_{\xi 2} & =\bar{e}_{\xi 3}+e_{1}^{1}, \\
\dot{\bar{e}}_{\xi 3} & =\bar{e}_{\xi 4}+\left(v_{1}^{1}-z_{2}^{1}\right) \\
& =-\Lambda_{3} \bar{e}_{\xi 3}-\Lambda_{2} \bar{e}_{\xi 2}-\Lambda_{1} \bar{e}_{\xi 1}+\sigma_{P}+\dot{e}_{1}^{1}-e_{2}^{1}, \\
\bar{e}_{\xi 4} & =-\Lambda_{3} \bar{e}_{\xi 3}-\Lambda_{2} \bar{e}_{\xi 2}-\Lambda_{1} \bar{e}_{\xi 1}+\sigma_{P} .
\end{aligned}
$$

A more compact form of dynamics (40)-(42) can be obtained:

$$
\dot{\bar{e}}_{\xi}=A \bar{e}_{\xi}+\bar{u},
$$

where $\bar{e}_{\xi}=\left[\begin{array}{lll}\bar{e}_{\xi 1} & \bar{e}_{\xi 2} & \bar{e}_{\xi 3}\end{array}\right]^{T}, \quad \bar{u}=\left[\begin{array}{lll}0 & e_{1}^{1} & \sigma_{P}+\dot{e}_{1}^{1}-e_{2}^{1}\end{array}\right]^{T}$, and its norm is bounded. The matrix $A$ can be expressed as

$$
A=\left[\begin{array}{ccc}
0 & I & 0 \\
0 & 0 & I \\
-\Lambda_{1} & -\Lambda_{2} & -\Lambda_{3}
\end{array}\right],
$$

which is a Hurwitz matrix. Let us define a finite-time bounded (FTB) function as

$$
V_{2}\left(\bar{e}_{\xi}\right)=\frac{1}{2} \bar{e}_{\xi}^{T} \bar{e}_{\xi}
$$

Considering the dynamics (44), the derivative of (46) can be obtained as

$$
\begin{aligned}
\dot{V}_{2}\left(\bar{e}_{\xi}\right) & =\bar{e}_{\xi}^{T} \dot{\bar{e}}_{\xi}=\bar{e}_{\xi}^{T}\left(A \bar{e}_{\xi}+\bar{u}\right)=\bar{e}_{\xi}^{T} A \bar{e}_{\xi}+\bar{e}_{\xi}^{T} \bar{u} \\
& \leq \lambda_{\max }(A) \bar{e}_{\xi}^{T} \bar{e}_{\xi}+\left(\frac{1}{2} \bar{e}_{\xi}^{T} \bar{e}_{\xi}+\frac{1}{2} \bar{u}^{T} \bar{u}\right) \\
& \leq\left[\lambda_{\max }(A)+\frac{1}{2}\right] \bar{e}_{\xi}^{T} \bar{e}_{\xi}+\frac{1}{2} \bar{u}^{T} \bar{u} \\
& \leq K_{v 2} V_{2}\left(\bar{e}_{\xi}\right)+L_{v 2},
\end{aligned}
$$

where $K_{v 2}=2 \lambda_{\max }(A)+1, L_{v 2}=1 / 2 \bar{u}^{T} \bar{u}$, and $\lambda_{\max }(A)$ represents the maximum eigenvalue of matrix $A$.

Therefore, we can derive the FTB function $V_{2}\left(\bar{e}_{\xi}\right)$, and so the variables $\bar{e}_{\xi}=\left[\begin{array}{lll}\bar{e}_{\xi 1} & \bar{e}_{\xi 2} & \bar{e}_{\xi 3}\end{array}\right]^{T}$ will not tend to infinity in any finite time. Moreover, according to equation (43), the variable $\bar{e}_{\xi 4}$ will not tend to infinity in any finite time either. In other words, the transition process of FTDO and the transition process of sliding mode dynamics do not destabilize the closed-loop helicopter system controlled by observerbased control laws.

In the third step, we will show that the variables $\bar{e}_{\xi 1}, \bar{e}_{\xi 2}$, $\bar{e}_{\xi 3}$, and $\bar{e}_{\xi 4}$ will converge to zero asymptotically.
Since the estimation errors $e_{i}^{j}$ will go to zero in a finite time $t_{f}$ and the sliding variable $\sigma_{P}$ will go to the origin in a finite time $t_{\sigma}$, the dynamics ((40)-(42) and (43)) will reduce to

$$
\begin{aligned}
& \dot{\bar{e}}_{\xi 1}=\bar{e}_{\xi 2}, \\
& \dot{\bar{e}}_{\xi_{2}}=\bar{e}_{\xi 3}, \\
& \dot{\bar{e}}_{\xi 3}=-\Lambda_{3} \bar{e}_{\xi 3}-\Lambda_{2} \bar{e}_{\xi 2}-\Lambda_{1} \bar{e}_{\xi 1}, \\
& \bar{e}_{\xi 4}=-\Lambda_{3} \bar{e}_{\xi 3}-\Lambda_{2} \bar{e}_{\xi 2}-\Lambda_{1} \bar{e}_{\xi 1},
\end{aligned}
$$

when $t>t_{\xi}$, where $t_{\xi}=\max \left\{t_{f}, t_{\sigma}\right\}$.

The matrix $A$ is a Hurwitz matrix, so the variables $\bar{e}_{\xi 1}, \bar{e}_{\xi 2}$, and $\bar{e}_{\xi_{3}}$ will converge to zero asymptotically. Furthermore, according to the definition of matrices $\Lambda_{1}, \Lambda_{2}$, and $\Lambda_{3}$, the variable $\bar{e}_{\xi 4}$ will converge to zero too.In summary, the position $P$ of the unmanned helicopter will converge to the desired trajectory asymptotically and all the states remain bounded despite the presence of matched disturbance $d_{2}$ and mismatched disturbance $d_{1}$.

\section{Simulation Results}

Some numerical simulation results are provided in this section to demonstrate the effectiveness of the proposed continuous sliding mode controller of the small-scale unmanned helicopter. Furthermore, in order to evaluate the superiority of the proposed CSMC method, both the traditional SMC and ENDO-SMC [28] are employed as comparative methods.

The parameters of the small-scale unmanned helicopter are given as follows. $m=8.2 \mathrm{~kg}, g=9.81 \mathrm{~m} \cdot \mathrm{s}^{-2}, Z_{w}=$ $-0.7615 \mathrm{~s}^{-1}, Z_{\mathrm{col}}=-131.4125 \mathrm{~m} \cdot \mathrm{rad}^{-1} \cdot \mathrm{s}^{-2}, J=\operatorname{diag}(0.18$, $0.34,0.28) \mathrm{kg} \cdot \mathrm{m}^{2}, \quad A=\operatorname{diag}(-48.1757,-25.5048,-0.9808)$ $\mathrm{s}^{-1}$, and

$$
B=\left[\begin{array}{cccc}
0 & 0 & 1689.5 & 0 \\
0 & 894.5 & 0 & 0 \\
-0.3705 & 0 & 0 & 135.8
\end{array}\right] \mathrm{s}^{-2} .
$$

The parameters of the controllers are chosen as follows. The coefficients of the FTDOs are $\lambda_{0}^{1}=4, \lambda_{1}^{1}=3$, $\lambda_{2}^{1}=3, \lambda_{3}^{1}=4, L_{1}=10, \lambda_{0}^{2}=4, \lambda_{1}^{2}=4, L_{2}=15, \lambda_{0}^{3}=3, \lambda_{1}^{3}=3$, and $L_{3}=10$. The coefficients of the sliding surface are $\Lambda_{1}=$ $\operatorname{diag}(27,27,27), \Lambda_{2}=\operatorname{diag}(9,9,9), \Lambda_{3}=\operatorname{diag}(1,1,1)$, and $\lambda_{4}=1$. The parameters of the continuous sliding mode controller are $K_{P}=\operatorname{diag}(5,15,20), K_{\psi}=2, \alpha_{1}=0.8$, and $\alpha_{2}=0.8$. The parameters of the ENDO-SMC are $K_{P}=$ diag $(5,25,40)$ and $K_{\psi}=2$. The parameters of the traditional SMC are $K_{P}=\operatorname{diag}(60,100,300)$ and $K_{\psi}=10$.

The lumped disturbances affecting the unmanned helicopter system are $\left[\begin{array}{ll}f_{d}^{T} & \tau_{d}^{T}\end{array}\right]^{T}=\Delta\left[\begin{array}{llll}P^{T} & V^{T} & \Theta^{T} & \Omega^{T}\end{array}\right]^{T}$ $+d_{\text {wind }}$, where $\Delta$ represents the model uncertainty matrix, and all the elements are pseudorandom values in the interval 

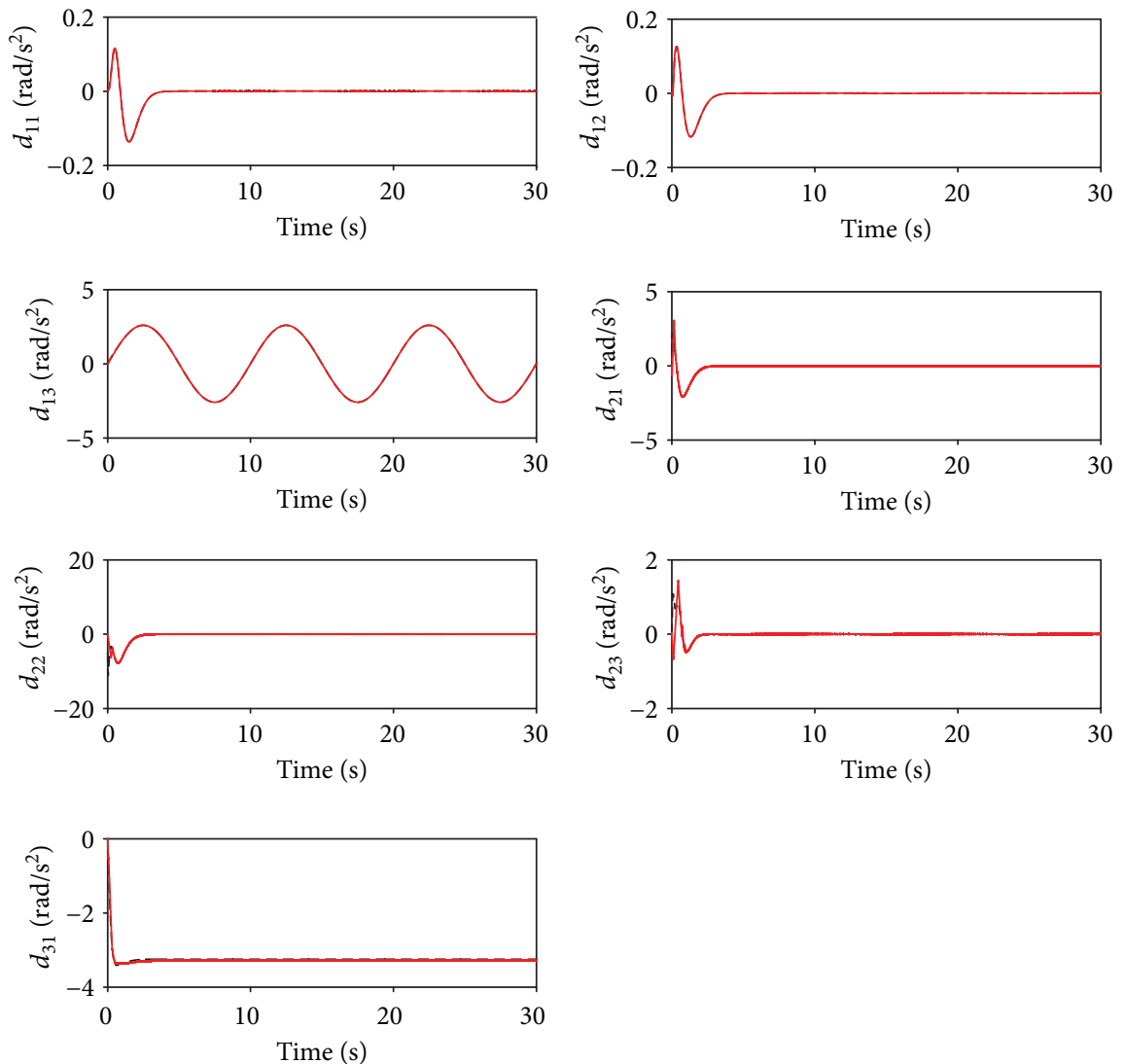

- - Real disturbances

- Estimated disturbances

FIgURE 3: Disturbance estimation of hovering.
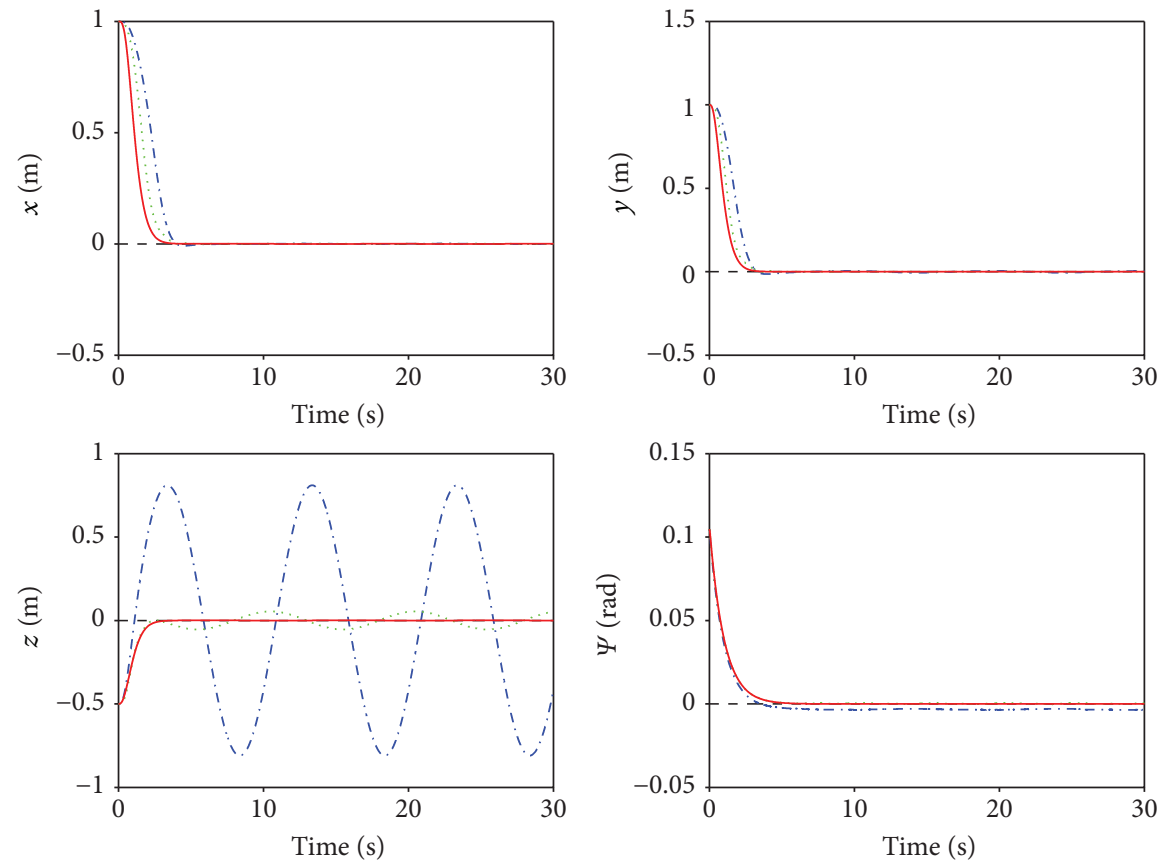

--- Reference $\quad \cdots$ ENDO -SMC

_.. Traditional SMC — Proposed CSMC

FIGURE 4: The response curves of position and yaw angle of hovering. 

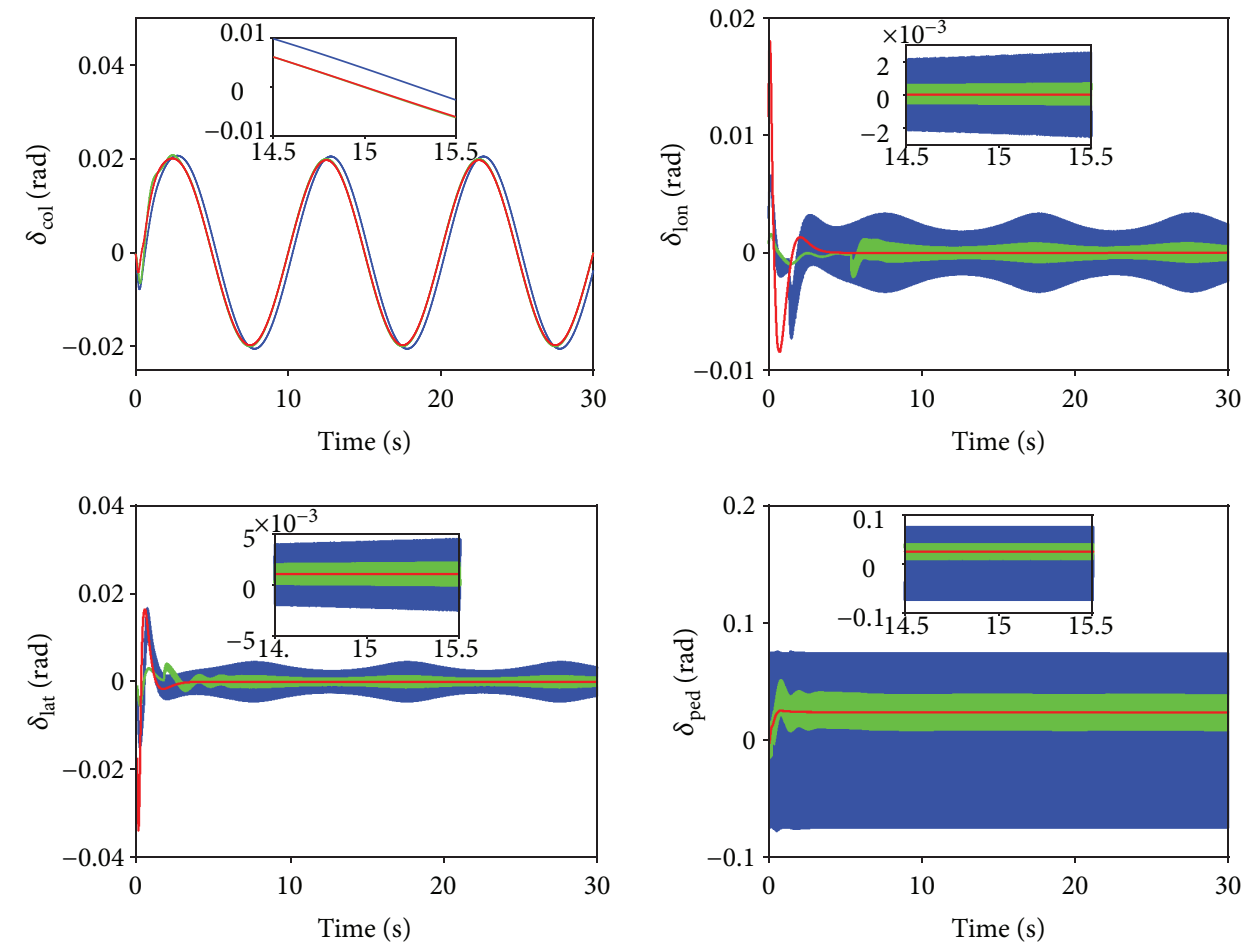

- Traditional SMC

— ENDO-SMC

— Proposed CSMC

FIGURE 5: The response curves of control inputs of hovering.
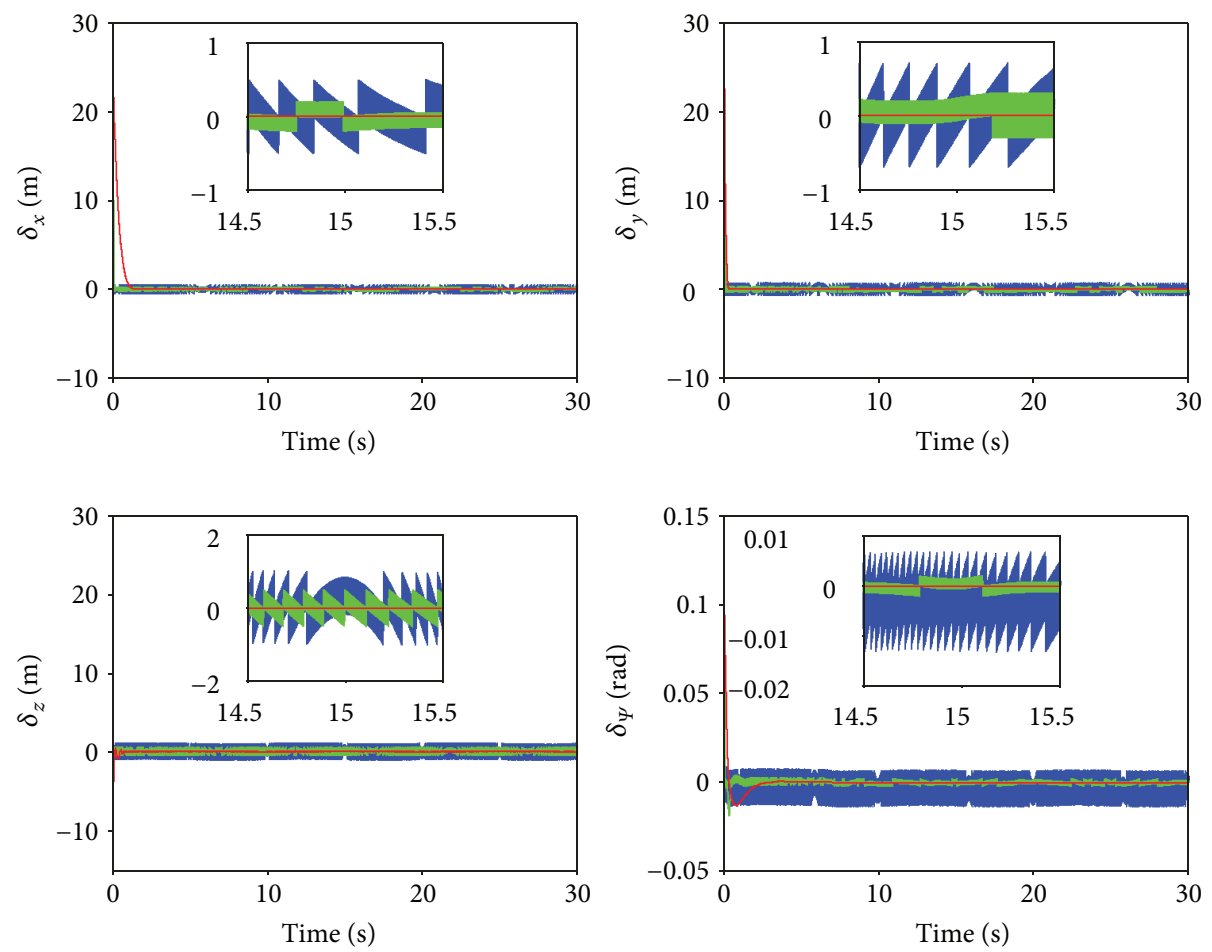

Traditional SMC

ENDO-SMC

— Proposed CSMC

FIgURE 6: The response curves of sliding surfaces of hovering. 

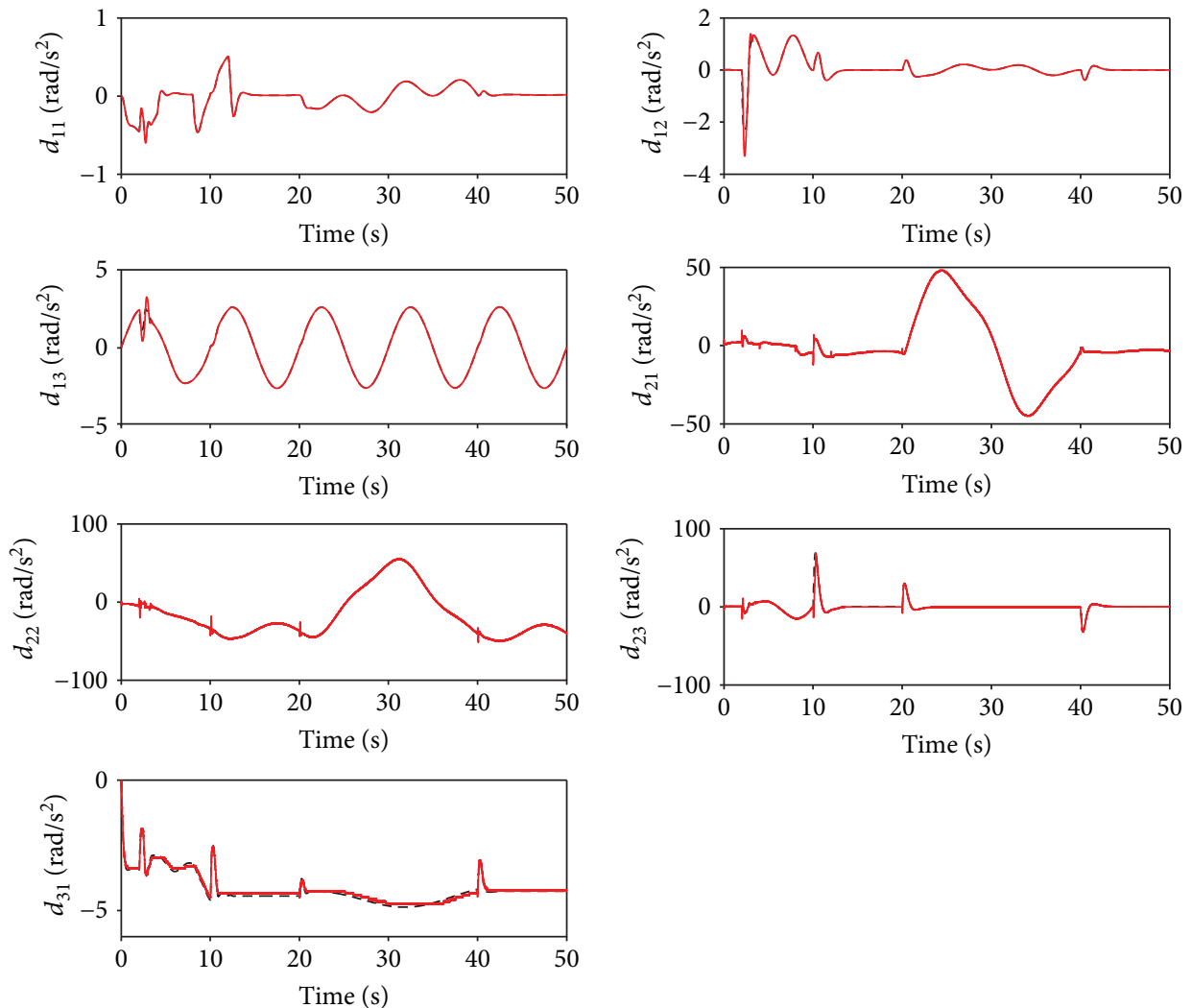

- - - Real disturbances

_ Estimated disturbances

FIGURE 7: Disturbance estimation of maneuver.
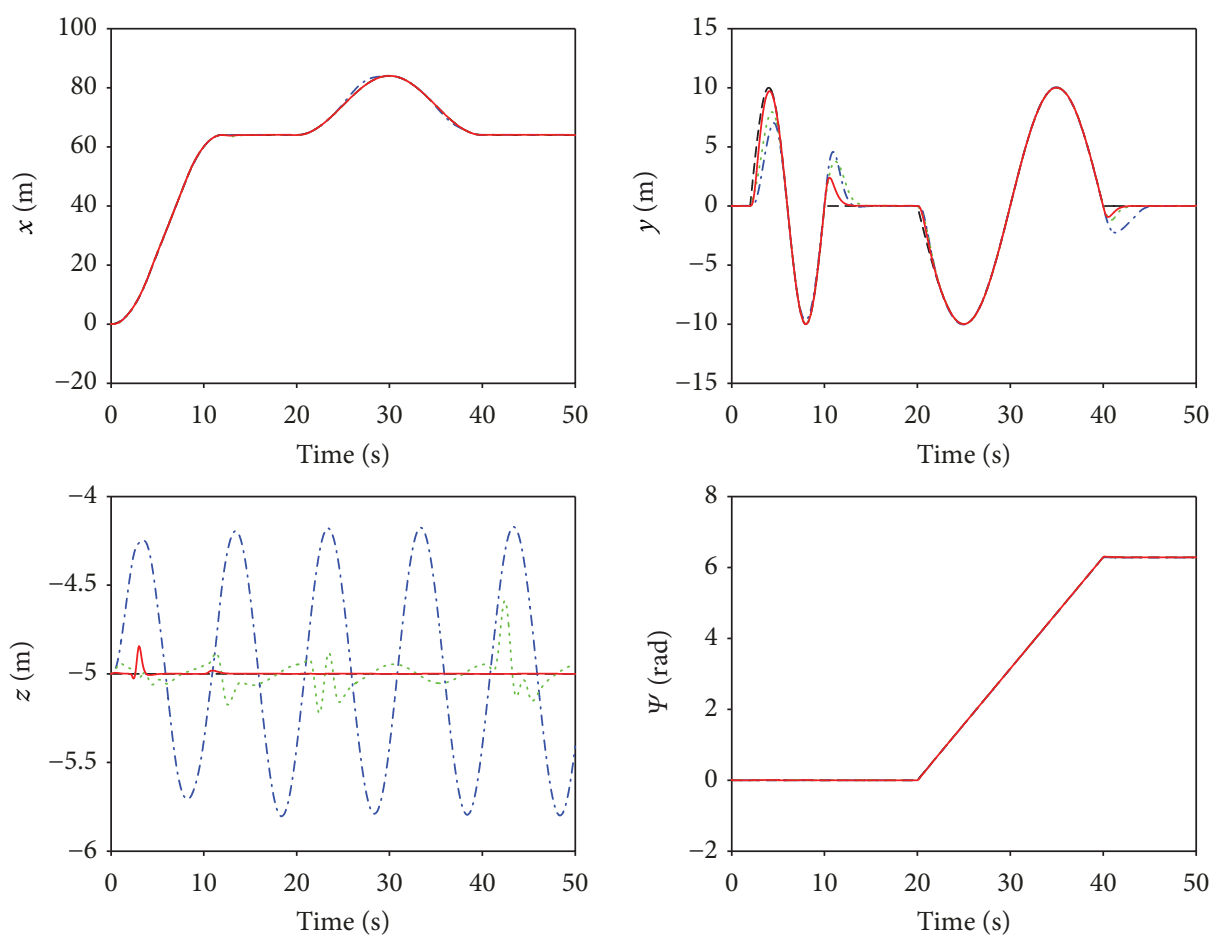

- - - Reference

ENDO-SMC

... Traditional SMC _ Proposed CSMC

Figure 8: The response curves of position and yaw angle of maneuver. 

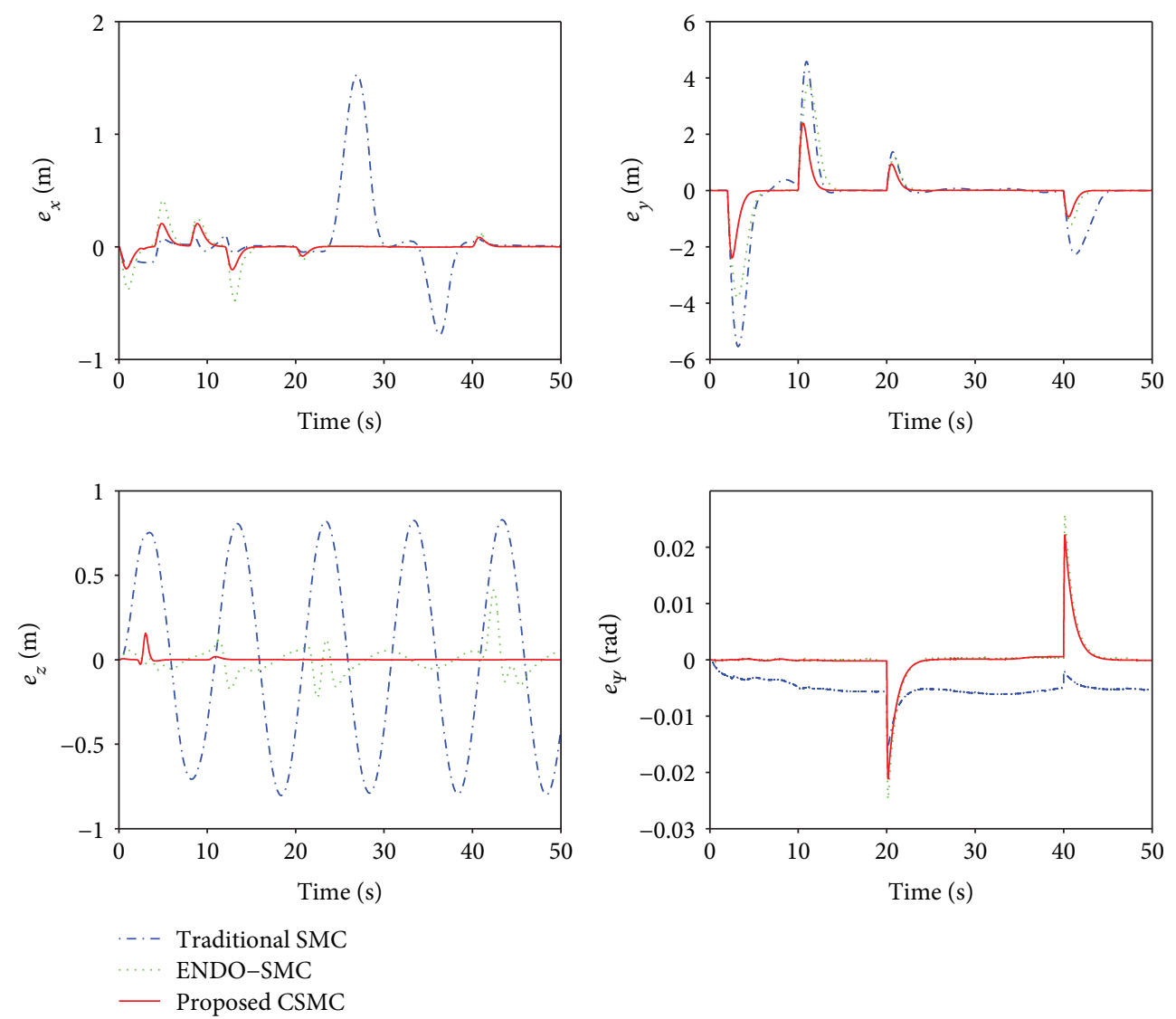

FIGURE 9: The response curves of tracking errors of maneuver.

of $(-1,1)$, and $d_{\text {wind }}=\left[\begin{array}{llllll}0 & 0 & f_{d w} & 0 & 0 & \tau_{d w}\end{array}\right]^{T}$ represents the external disturbances produced by wind gusts [39].

In order to examine the performance of the proposed flight controller comprehensively, two types of flight simulation are conducted here.

5.1. Hovering Flight Simulation. To validate the hovering performance of the unmanned helicopter, a hovering flight simulation is carried out. The initial position and yaw angle of the helicopter are $P_{0}=\left[\begin{array}{lll}1 & 1 & -0.5\end{array}\right]^{T} \mathrm{~m}$ and $\psi_{0}=$ $(\pi / 30) \mathrm{rad}$, respectively. The other state variables are all set to zero. The control goal of this hovering flight simulation is to stabilize the helicopter to the origin $P_{r}=\left[\begin{array}{lll}0 & 0 & 0\end{array}\right]^{T} \mathrm{~m}$ and $\psi_{r}=0 \mathrm{rad}$ in the presence of both matched and mismatched disturbances.

The simulation results are illustrated in Figures 3-6. Figure 3 shows the estimates of the disturbances of hovering by FTDOs. The response curves of the position and yaw angle are depicted in Figure 4. It can be seen that all the positions of $x, y$, and $z$ can be kept in the origin precisely under the proposed CSMC method. The position of $x$ and $y$ can almost be kept in the origin under the traditional SMC and ENDO-SMC methods. However, the position of $z$ results in fluctuation severely under the traditional SMC method, and it suffers from fluctuation to a certain degree under the ENDO-SMC method too. As shown in Figure 3, the mismatched disturbance $d_{1}{ }^{(3)}$ affecting the $z$-axis is much larger

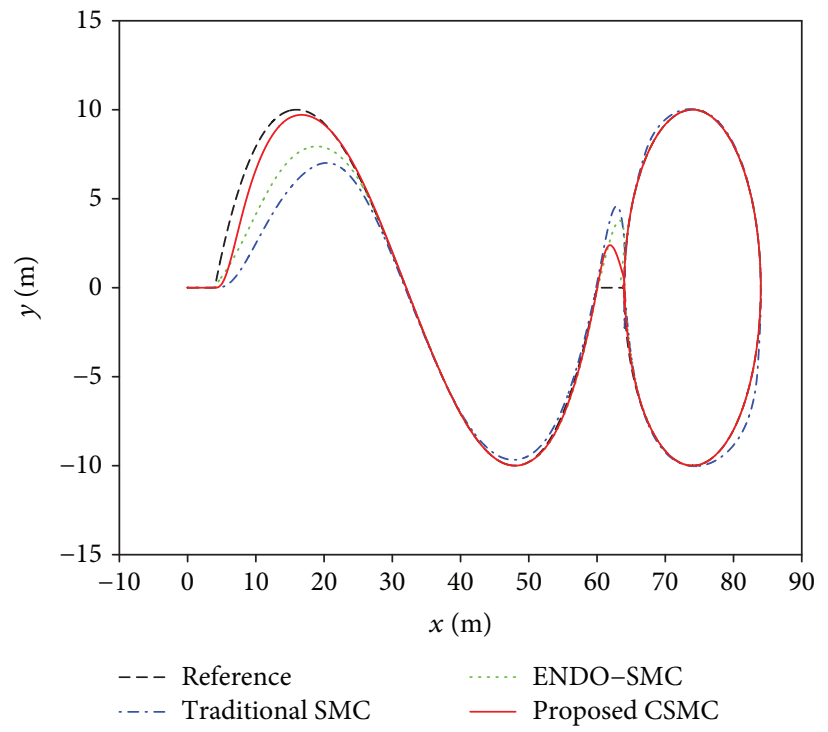

Figure 10: $x-y$ plane of maneuver.

than $d_{1}{ }^{(1)}$ and $d_{1}{ }^{(2)}$ affecting the $x$ - and $y$-axes, which reveals that the proposed CSMC method possesses much stronger ability to suppress mismatched disturbances. Furthermore, the yaw angle $\psi$ can stay near zero under all the three control methods; for that, the yaw dynamics is only subjected to matched disturbance. Figure 5 depicts the response 

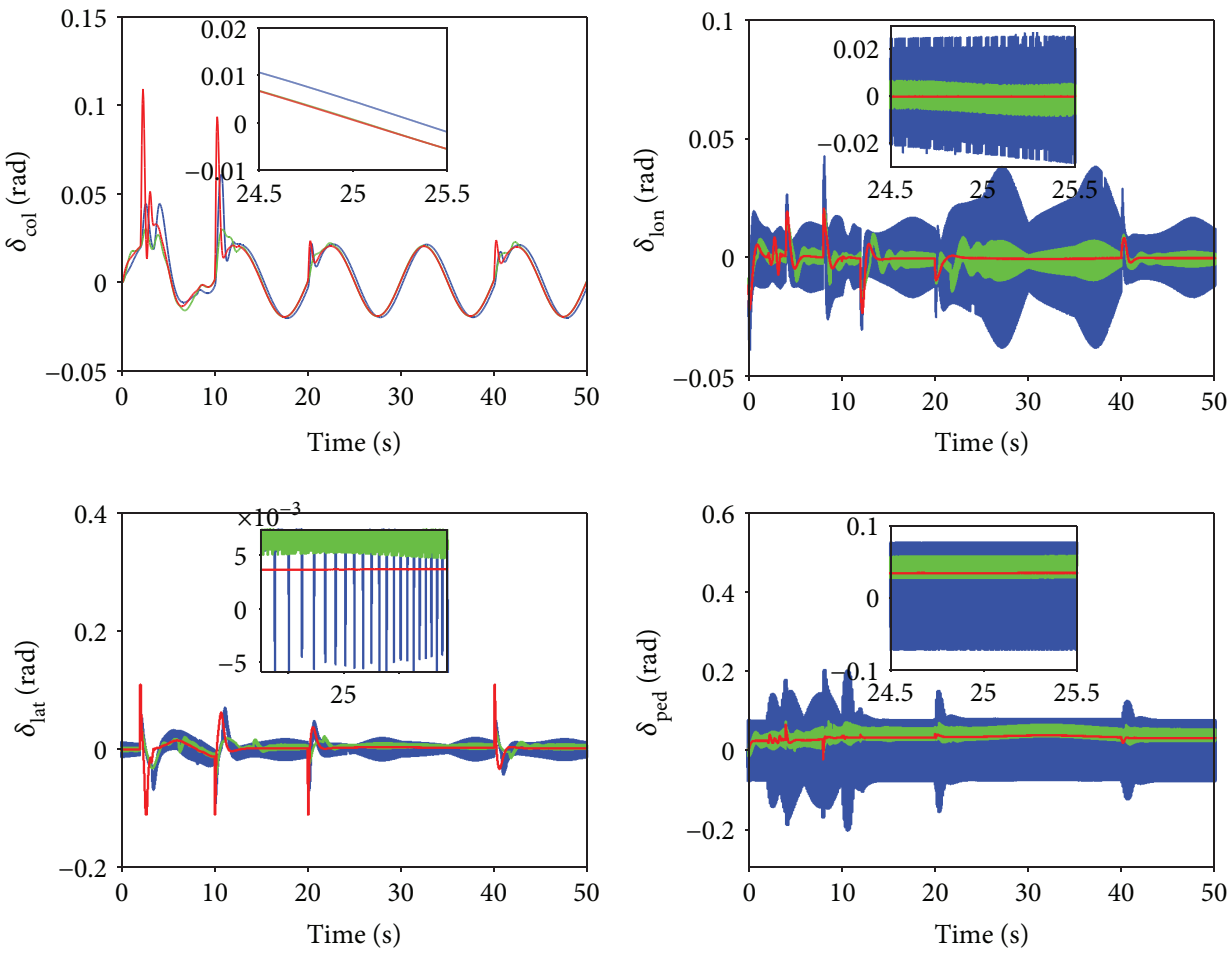

— Traditional SMC

ENDO-SMC

— Proposed CSMC

FIGURE 11: The response curves of control inputs of maneuver.
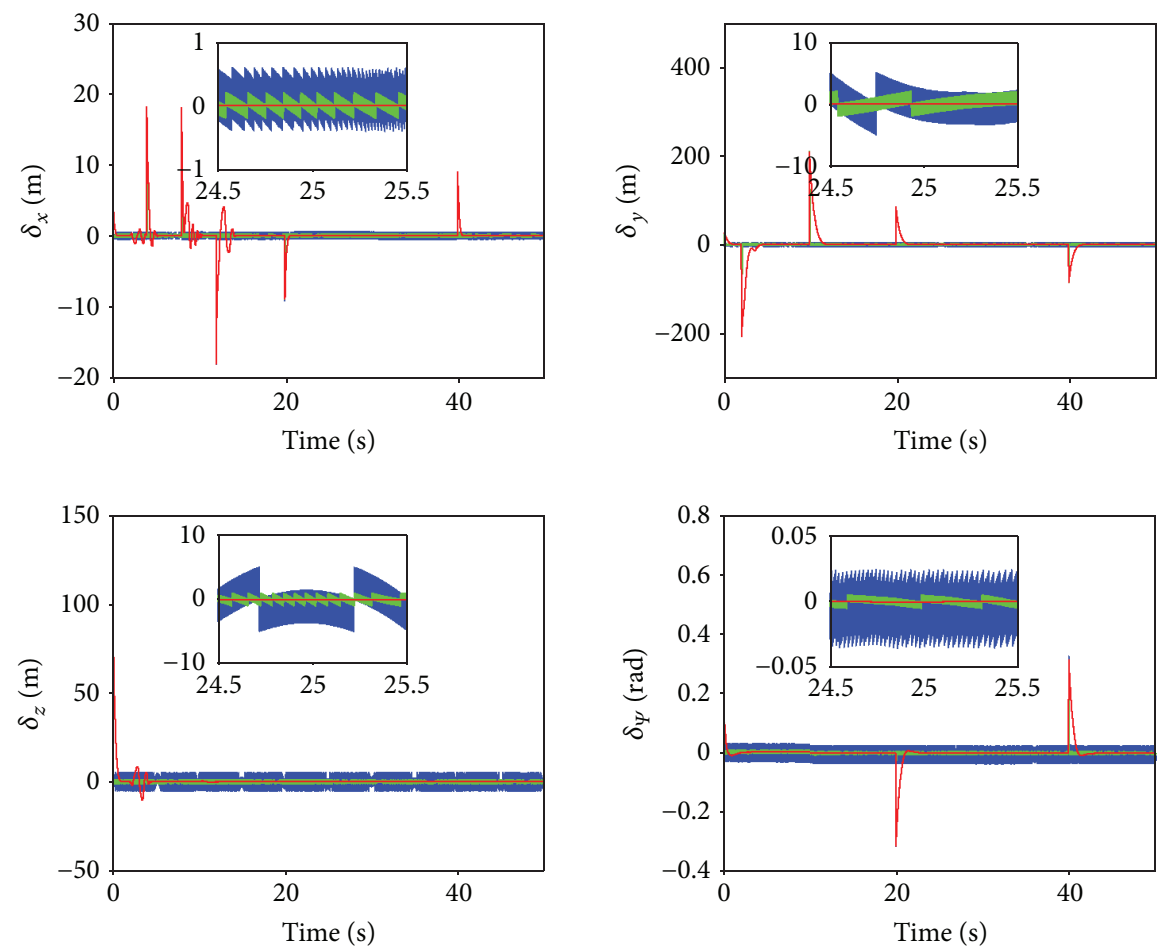

Traditional SMC

ENDO-SMC

— Proposed CSMC

Figure 12: The response curves of sliding surfaces of maneuver. 
curves of the control inputs. Figure 6 shows the response curves of the sliding surfaces. Both the traditional SMC and ENDO-SMC methods are suffering from a chattering phenomenon due to the discontinuous switching control actions. On the other hand, from these two figures, we can obtain that the proposed CSMC method does not lead to any chattering phenomenon.

5.2. Maneuver Flight Simulation. To validate the tracking performance of the unmanned helicopter, a maneuver flight simulation is carried out.

A comprehensive maneuver flight trajectory is given as [7]

$$
\begin{aligned}
& x_{r}(t)= \begin{cases}t^{2}, & t \leq 4 s, \\
8 t-16, & 4<t \leq 8 s, \\
-t^{2}+24 t-80, & 8<t \leq 12 s, \\
64, & 12<t \leq 20 s, \\
74+10 \cos \left(\frac{\pi}{10}(t-20)-\pi\right), & 20<t \leq 40 s, \\
64, & t>40 s,\end{cases} \\
& y_{r}(t)= \begin{cases}0, & t \leq 2 s, \\
10 \sin \left(\frac{\pi}{4}(t-2)\right), & 2<t \leq 10 s, \\
0, & 10<t \leq 20 s \\
10 \sin \left(\frac{\pi}{10}(t-20)-\pi\right), & 20<t \leq 40 s, \\
0, & t>40 s,\end{cases} \\
& z_{r}(t)=-5, \quad t \leq 0 s, \\
& \psi_{r}(t)= \begin{cases}0, & t \leq 20 s, \\
0.1 \pi(t-20), & 20<t \leq 40 s, \\
2 \pi, & t>40 s .\end{cases}
\end{aligned}
$$

The initial values of the state variables are all set to zero.

The simulation results are illustrated in Figures 7-12. Figure 7 depicts the estimates of the disturbances of the maneuver flight by FTDOs. Figure 8 shows the response curves of the position and yaw angle. We can see that the control performance of the proposed CSMC method is better than those of the traditional SMC and ENDO-SMC methods due to its more precise tracking performance and gentler dynamic process. Figure 9 shows the tracking errors of the maneuver flight. It can be observed that the tracking errors of the proposed control method are smaller than those of the two control methods, especially for the tracking error of position $z$. In addition, Table 1 gives the root mean square (RMS) of the tracking errors, which supports the aforementioned analysis primely. The $x-y$ plane of the maneuver is illustrated in Figure 10, which shows the horizontal trajectory intuitively. Figure 11 depicts the response curves of the
TABLE 1: The root mean square (RMS) of tracking errors.

\begin{tabular}{lcccc}
\hline Method & Position $x$ & Position $y$ & Position $z$ & Yaw angle $\psi$ \\
\hline Proposed CSMC & $0.0578 \mathrm{~m}$ & $0.4651 \mathrm{~m}$ & $0.0114 \mathrm{~m}$ & $0.0033 \mathrm{rad}$ \\
ENDO-SMC & $0.1114 \mathrm{~m}$ & $0.9403 \mathrm{~m}$ & $0.0816 \mathrm{~m}$ & $0.0039 \mathrm{rad}$ \\
Traditional SMC & $0.3614 \mathrm{~m}$ & $1.2816 \mathrm{~m}$ & $0.5622 \mathrm{~m}$ & $0.0054 \mathrm{rad}$ \\
\hline
\end{tabular}

TABLE 2: The root mean square (RMS) of control inputs.

\begin{tabular}{lcccc}
\hline Method & Input $\delta_{\text {col }}$ & Input $\delta_{\text {lon }}$ & Input $\delta_{\text {lat }}$ & Input $\delta_{\text {ped }}$ \\
\hline Proposed CSMC & $0.0148 \mathrm{rad}$ & $0.0036 \mathrm{rad}$ & $0.0099 \mathrm{rad}$ & $0.0229 \mathrm{rad}$ \\
ENDO-SMC & $0.0172 \mathrm{rad}$ & $0.0051 \mathrm{rad}$ & $0.0159 \mathrm{rad}$ & $0.0435 \mathrm{rad}$ \\
Traditional SMC & $0.0187 \mathrm{rad}$ & $0.0155 \mathrm{rad}$ & $0.0215 \mathrm{rad}$ & $0.0789 \mathrm{rad}$ \\
\hline
\end{tabular}

control inputs. Figure 12 shows the response curves of the sliding surfaces. It can be noted that the control inputs and the sliding surfaces of the proposed CSMC method are continuous and free from any chattering phenomenon. Furthermore, the RMS of the control inputs is given in Table 2. It can be seen that the control energy of the proposed control method is lower than that of the other two control methods, which means that the proposed CSMC method performs the flight mission most effectively.

\section{Conclusion}

In this paper, a novel continuous sliding mode controller based on the approximate feedback linearization and FTDO is developed for the small-scale unmanned helicopter with matched and mismatched disturbances. The CSMC method is robust to both matched and mismatched disturbances and does not result in any chattering phenomenon. Furthermore, representative simulation results demonstrate that the proposed CSMC method exhibits superior control performance compared with the traditional SMC and ENDOSMC methods. Additionally, future works include the experiment tests for the proposed control method on an experimental helicopter platform.

\section{Data Availability}

The data used to support the findings of this study are included within the article.

\section{Conflicts of Interest}

The authors declare that there is no conflict of interest.

\section{Acknowledgments}

This work is supported by the National Natural Science Foundation of China under grants 61803182 and 61703118 and the Natural Science Foundation of Jiangsu Province under grant BK20180593. 


\section{References}

[1] F. Chen, L. Cai, B. Jiang, and G. Tao, "Direct self-repairing control for a helicopter via quantum multi-model and disturbance observer," International Journal of Systems Science, vol. 47, no. 3, pp. 533-543, 2014.

[2] X. Fang, A. Wu, Y. Shang, and N. Dong, "Robust control of small-scale unmanned helicopter with matched and mismatched disturbances," Journal of the Franklin Institute, vol. 353, no. 18, pp. 4803-4820, 2016.

[3] J. Hu and H. Gu, "Survey on flight control technology for large-scale helicopter," International Journal of Aerospace Engineering, vol. 2017, Article ID 5309403, 14 pages, 2017.

[4] F. Leonard, A. Martini, and G. Abba, "Robust nonlinear controls of model-scale helicopters under lateral and vertical wind gusts," IEEE Transactions on Control Systems Technology, vol. 20, no. 1, pp. 154-163, 2012.

[5] H. Kim, "A flight control system for aerial robots: algorithms and experiments," Control Engineering Practice, vol. 11, no. 12, pp. 1389-1400, 2003.

[6] M. V. Kumar, P. Sampath, S. Suresh, S. N. Omkar, and R. Ganguli, "Design of a stability augmentation system for a helicopter using LQR control and ADS-33 handling qualities specifications," Aircraft Engineering and Aerospace Technology, vol. 80, no. 2, pp. 111-123, 2008.

[7] G. W. Cai, B. M. Chen, and T. H. Lee, Unmanned Rotorcraft Systems, Springer, Heidelberg, 2010.

[8] S. Zhao, B. Huang, and Y. S. Shmaliy, "Bayesian state estimation on finite horizons: the case of linear state-space model," Automatica, vol. 85, pp. 91-99, 2017.

[9] T. J. Koo and S. Sastry, "Output tracking control design of a helicopter model based on approximate linearization," in Proceedings of 37th IEEE decision and control, pp. 3635-3640, Tampa, FL, USA, 1998.

[10] J. Hu and H. Zhang, "Immersion and invariance based command-filtered adaptive backstepping control of VTOL vehicles," Automatica, vol. 49, no. 7, pp. 2160-2167, 2013.

[11] B. Zhao, B. Xian, Y. Zhang, and X. Zhang, "Nonlinear robust sliding mode control of a quadrotor unmanned aerial vehicle based on immersion and invariance method," International Journal of Robust and Nonlinear Control, vol. 25, no. 18, pp. 3714-3731, 2015.

[12] W.-H. Chen, D. J. Ballance, P. J. Gawthrop, and J. O'Reilly, “A nonlinear disturbance observer for robotic manipulators," IEEE Transactions on Industrial Electronics, vol. 47, no. 4, pp. 932-938, 2000.

[13] Z. Gao, "On the centrality of disturbance rejection in automatic control," ISA Transactions, vol. 53, no. 4, pp. 850-857, 2014.

[14] A. Mujumdar, B. Tamhane, and S. Kurode, "Observer-based sliding mode control for a class of noncommensurate fractional-order systems," IEEE/ASME Transactions on Mechatronics, vol. 20, no. 5, pp. 2504-2512, 2015.

[15] B. Tamhane and S. Kurode, "Finite time state and disturbance estimation for robust performance of motion control systems using sliding modes," International Journal of Control, vol. 91, no. 5, pp. 1171-1182, 2017.

[16] W. Xue, R. Madonski, K. Lakomy, Z. Gao, and Y. Huang, "Add-on module of active disturbance rejection for set-point tracking of motion control systems," IEEE Transactions on Industry Applications, vol. 53, no. 4, pp. 4028-4040, 2017.
[17] Y. Huang and W. Messner, "A novel disturbance observer design for magnetic hard drive servo system with a rotary actuator," IEEE Transactions on Magnetics, vol. 34, no. 4, pp. 1892 1894, 1998.

[18] C. Ming, R. Sun, and X. Wang, "Velocity control based on active disturbance rejection for air-breathing supersonic vehicles," Complexity, vol. 2018, Article ID 6217657, 11 pages, 2018.

[19] L. Sun, D. Li, and K. Y. Lee, "Enhanced decentralized PI control for fluidized bed combustor via advanced disturbance observer," Control Engineering Practice, vol. 42, pp. 128-139, 2015.

[20] H. K. Khalil, Nonlinear Systems, Prentice Hall, New Jersey, 2002.

[21] C. Peng, J. Fang, and X. Xu, "Mismatched disturbance rejection control for voltage-controlled active magnetic bearing via state-space disturbance observer," IEEE Transactions on Power Electronics, vol. 30, no. 5, pp. 2753-2762, 2015.

[22] J. Yang, W. H. Chen, S. Li, L. Guo, and Y. Yan, "Disturbance/uncertainty estimation and attenuation techniques in PMSM drives-a survey," IEEE Transactions on Industrial Electronics, vol. 64, no. 4, pp. 3273-3285, 2017.

[23] S. Li, H. Sun, J. Yang, and X. Yu, "Continuous finite-time output regulation for disturbed systems under mismatching condition," IEEE Transactions on Automatic Control, vol. 60, no. 1, pp. 277-282, 2015.

[24] J. Yang, A. Zolotas, W. H. Chen, K. Michail, and S. Li, "Robust control of nonlinear MAGLEV suspension system with mismatched uncertainties via DOBC approach," ISA Transactions, vol. 50, no. 3, pp. 389-396, 2011.

[25] J. Yang, S. Li, and X. Yu, "Sliding-mode control for systems with mismatched uncertainties via a disturbance observer," IEEE Transactions on Industrial Electronics, vol. 60, no. 1, pp. 160-169, 2013.

[26] B. Tamhane, A. Mujumdar, and S. Kurode, "Mismatched disturbance compensation using sliding mode control," in 10th Asian Control Conference (ASCC), pp. 1-6, Kota Kinabalu, Malaysia, May 2015.

[27] J. Yang, J. Su, S. Li, and X. Yu, "High-order mismatched disturbance compensation for motion control systems via a continuous dynamic sliding-mode approach," IEEE Transactions on Industrial Informatics, vol. 10, no. 1, pp. 604-614, 2014.

[28] X. Fang, A. Wu, Y. Shang, and N. Dong, "A novel sliding mode controller for small-scale unmanned helicopters with mismatched disturbance," Nonlinear Dynamics, vol. 83, no. 1-2, pp. 1053-1068, 2016.

[29] J. J. Xiong and G. B. Zhang, "Global fast dynamic terminal sliding mode control for a quadrotor UAV," ISA Transactions, vol. 66, pp. 233-240, 2017.

[30] L. He, Y. J. Wu, and J. X. Zuo, "Sliding mode controller design for UAV based on backstepping control," in IEEE 2016 Chinese Guidance, Navigation and Control Conference (CGNCC), pp. 1448-1453, Nanjing, China, 2017.

[31] B. Xian, G. Jianchuan, Z. Yao, and Z. Bo, "Sliding mode tracking control for miniature unmanned helicopters," Chinese Journal of Aeronautics, vol. 28, no. 1, pp. 277-284, 2015.

[32] B. Zhu, "Nonlinear adaptive neural network control for a model-scaled unmanned helicopter," Nonlinear Dynamics, vol. 78, no. 3, pp. 1695-1708, 2014.

[33] M. Wan, M. Chen, and K. Yan, "Adaptive sliding mode tracking control for unmanned autonomous helicopters based on 
neural networks," Complexity, vol. 2018, Article ID 7379680, 11 pages, 2018.

[34] C. Liu, W.-H. Chen, and J. Andrews, "Tracking control of small-scale helicopters using explicit nonlinear MPC augmented with disturbance observers," Control Engineering Practice, vol. 20, no. 3, pp. 258-268, 2012.

[35] J. J. E. Slotine and W. P. Li, Applied Nonlinear Control, Prentice Hall, Englewood Cliffs, 1991.

[36] A. Levant, "Higher-order sliding modes, differentiation and output-feedback control," International Journal of Control, vol. 76, no. 9-10, pp. 924-941, 2003.

[37] Y. B. Shtessel, I. A. Shkolnikov, and A. Levant, "Smooth second-order sliding modes: missile guidance application," Automatica, vol. 43, no. 8, pp. 1470-1476, 2007.

[38] S. Li and Y. P. Tian, "Finite-time stability of cascaded timevarying systems," International Journal of Control, vol. 80, no. 4, pp. 646-657, 2007.

[39] A. Martini, F. Leonard, and G. Abba, "Dynamic modelling and stability analysis of model-scale helicopters under wind gust," Journal of Intelligent and Robotic Systems, vol. 54, no. 4, pp. 647-686, 2009. 


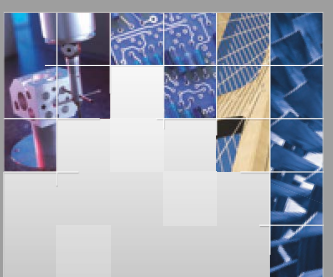

\section{Enfincering}
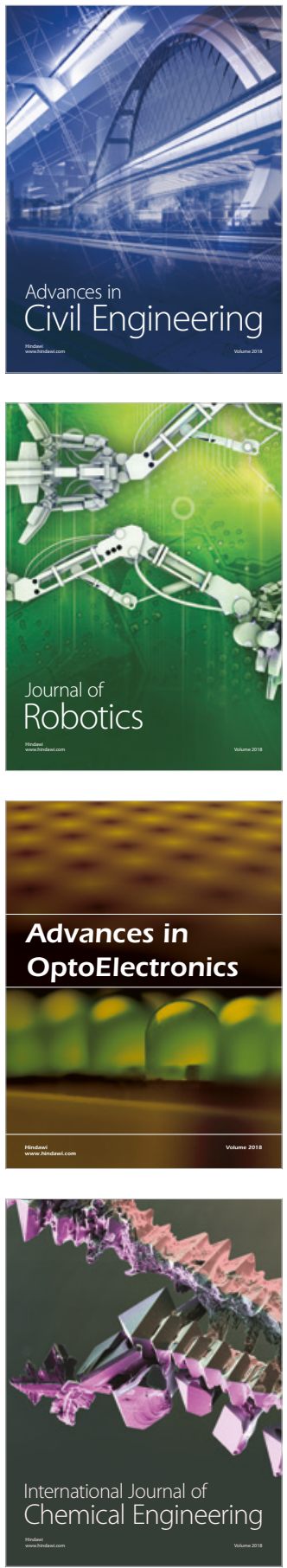

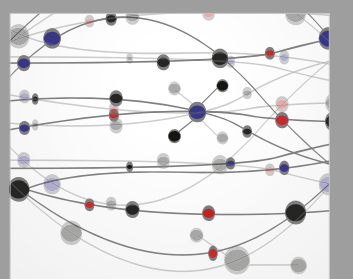

\section{Rotating \\ Machinery}

The Scientific World Journal

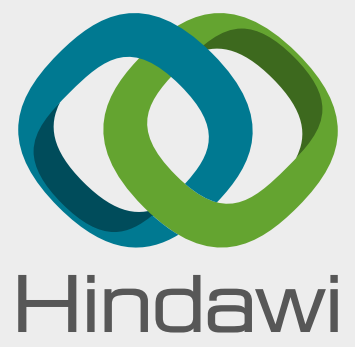

Submit your manuscripts at

www.hindawi.com
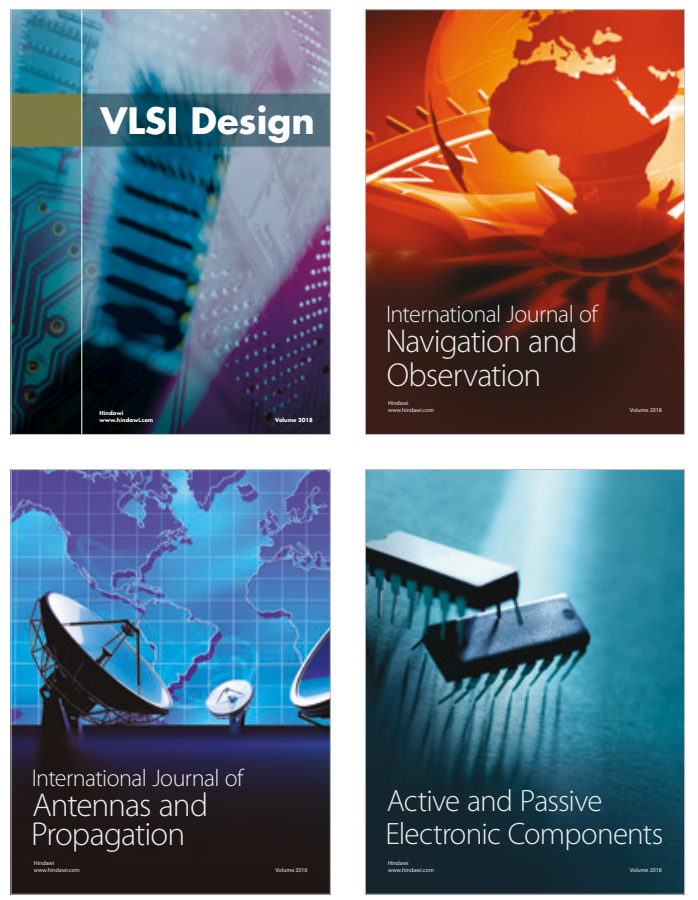
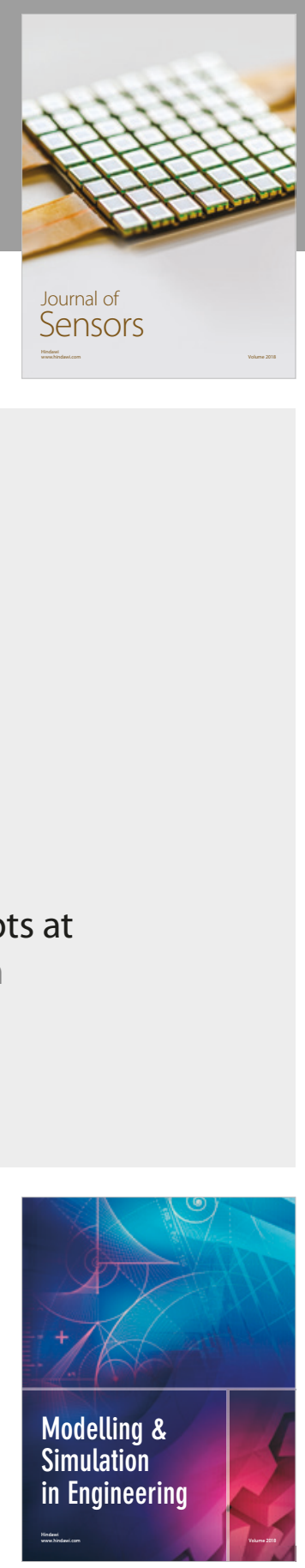

\section{Advances \\ Multimedia}
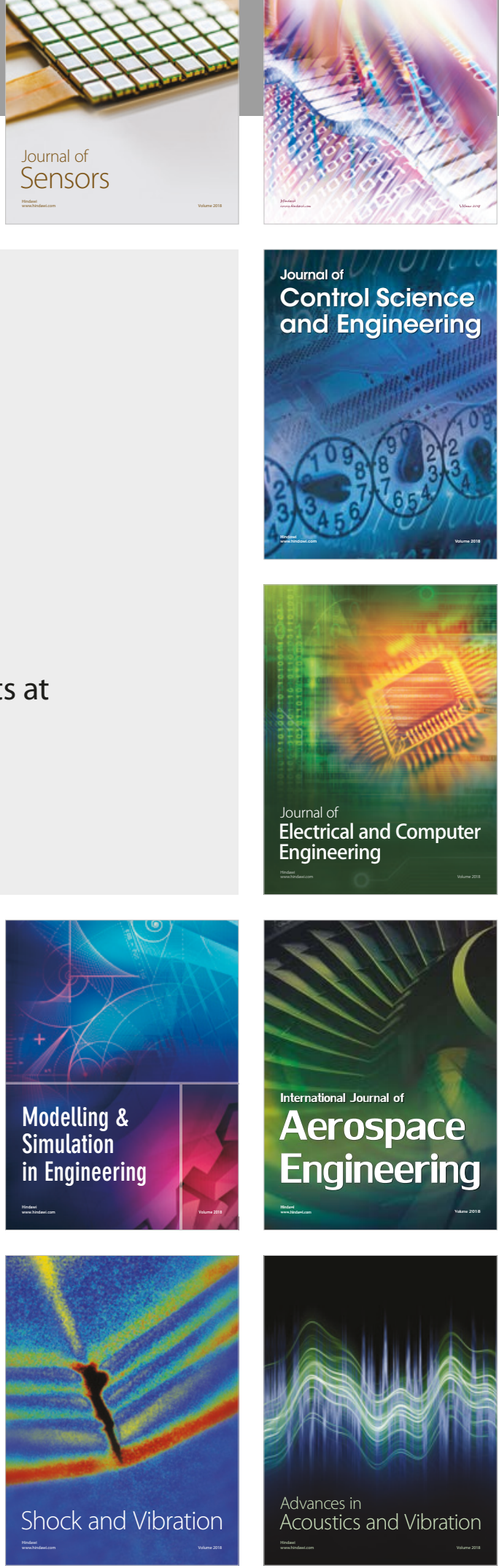